\title{
Perturbing rational harmonic functions by poles
}

\author{
Olivier Sète, Robert Luce, Jörg Liesen
}

November 3, 2018

\begin{abstract}
We study how adding certain poles to rational harmonic functions of the form $R(z)-\bar{z}$, with $R(z)$ rational and of degree $d \geq 2$, affects the number of zeros of the resulting functions. Our results are motivated by and generalize a construction of Rhie derived in the context of gravitational microlensing (arXiv:astro-ph/0305166). Of particular interest is the construction and the behavior of rational functions $R(z)$ that are extremal in the sense that $R(z)-\bar{z}$ has the maximal possible number of $5(d-1)$ zeros.
\end{abstract}

\section{Introduction}

This work is concerned with the zeros of functions in the complex variable $z$ of the form $R(z)-\bar{z}$, where $R(z)$ is a rational function. The analysis of such rational harmonic functions has received considerable attention in recent years. As nicely explained in the expository article of Khavinson and Neumann [12], they have important applications in gravitational microlensing; see also the survey [18]. In addition they are related to the matrix theory problem of expressing certain adjoints of a diagonalizable matrix as a rational function in the matrix [15].

In the sequel, whenever we write a rational function $R(z)=\frac{p(z)}{q(z)}$, we assume that the polynomials $p(z)$ and $q(z)$ are coprime, i.e., that they have no common zero. Then the degree of $R(z)$, denoted by $\operatorname{deg}(R)$, is defined as the maximum of the degrees of $p(z)$ and $q(z)$.

It is easy to see that $R(z)-\bar{z}$ has exactly one zero if $\operatorname{deg}(R)=0$, and either $0,1,2$ or infinitely many zeros (given by all points of a line or a circle in the complex plane) if $\operatorname{deg}(R)=1$. More interesting is the case $\operatorname{deg}(R)=d \geq 2$, which we consider in this paper. An important result of Khavinson and Neumann states that in this case $R(z)-\bar{z}$ may have at most $5(d-1)$ zeros [11]. Prior to the work of Khavinson and Neumann the astrophysicist Sun Hong Rhie (1955-2013) had constructed, in the context of gravitational lensing, examples of functions $R(z)-\bar{z}$ with exactly $5(d-1)$ zeros for every $d \geq 2[19]$. Hence the bound of Khavinson and Neumann is sharp for every $d \geq 2$. 
Motivated by the result of Khavinson and Neumann we call a rational function $R(z)$ of degree $d \geq 2$ extremal, when the function $R(z)-\bar{z}$ has the maximal possible number of $5(d-1)$ zeros. Examples of such extremal rational functions in the published literature are rare. For $d=2$ an example is given in [11]. The only other published example we are aware of is given by the construction of Rhie [19] and in the closely related works [2, 3]; see also [16]. Rhie's construction served as a motivation for our work, and some of our results can be considered a generalization of her original idea.

To briefly explain this idea, consider the rational harmonic function

$$
R_{0}(z)-\bar{z}, \quad \text { where } \quad R_{0}(z)=\frac{z^{d-1}}{z^{d}-r^{d}}
$$

and $r>0$ is a real parameter. For $d=2$ a straightforward computation shows that $R_{0}(z)-\bar{z}$ has 5 zeros if $r<1$, and fewer zeros for $r \geq 1$. Moreover, it was shown in [17] that for $d \geq 3$ the function $R_{0}(z)-\bar{z}$ has $3 d+1$ zeros if $r<\left(\frac{d-2}{d}\right)^{\frac{1}{2}}\left(\frac{2}{d-2}\right)^{\frac{1}{d}}$, and fewer zeros for larger values of $r$. Thus, $R_{0}(z)$ is extremal only for $d=2$ or $d=3$ (and when $r$ is small enough). Rhie suggested in [19 to perturb $R_{0}(z)-\bar{z}$ by adding a pole at one of its zeros, namely at the point $z=0$. More precisely, she showed that for a particular value $r>0$ and sufficiently small $\varepsilon>0$ the rational harmonic function

$$
R_{\varepsilon}(z)-\bar{z}, \quad \text { where } \quad R_{\varepsilon}(z)=(1-\varepsilon) R_{0}(z)+\frac{\varepsilon}{z},
$$

has $5 d$ zeros, so that $R_{\varepsilon}(z)$ (of degree $d+1$ ) is indeed extremal. An elementary proof and sharp bounds on the parameters $r$ and $\varepsilon$ that guarantee extremality of $R_{\varepsilon}(z)$ are given in [16].

A numerical example for Rhie's construction is shown in Figure 1, where we use phase portraits for visualization [23, 22]; see also Section 4 below. Here $d=\operatorname{deg}\left(R_{0}\right)=7$, and $r>0$ is chosen sufficiently small. Figure 1(a) shows that $R_{0}(z)-\bar{z}$ has $3 d+1=22$ zeros and 7 poles. We choose $\varepsilon=0.15$, which is sufficiently small in order to obtain an extremal function $R_{\varepsilon}(z)$, i.e. a function $R_{\varepsilon}(z)-\bar{z}$ with $5 d=35$ zeros; see Figure 1(b). Two essential observations are to be made when comparing Figures 1(a) and 1(b)

(1) The zero of $R_{0}(z)-\bar{z}$ at the perturbation point $z=0$ becomes a pole of the perturbed function, but all other zeros of $R_{0}(z)-\bar{z}$ "survive" the perturbation in the sense that perturbed versions of them are still zeros of $R_{\varepsilon}(z)-\bar{z}$.

(2) In a neighborhood of the perturbation point $z=0$ the function $R_{\varepsilon}(z)-$ $\bar{z}$ has $2 d$ additional zeros, which are located on two circles of radius approximately $\sqrt{\varepsilon}$ around $z=0$. (In Figure 1(b) the two circles are visually almost indistinguishable.)

While the function $R_{\varepsilon}(z)$ considered by Rhie arises from a convex combination of $R_{0}(z)$ with the pole $\frac{1}{z}$, it was shown in [16] that qualitatively the 


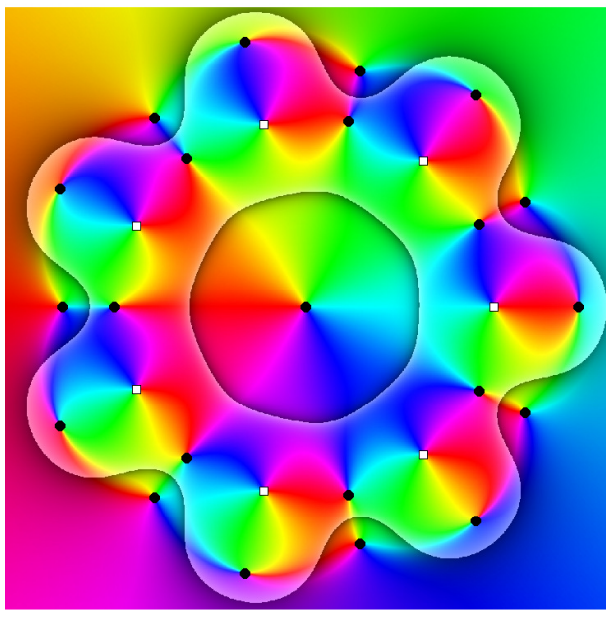

(a) Phase portrait of $R_{0}(z)-\bar{z}$

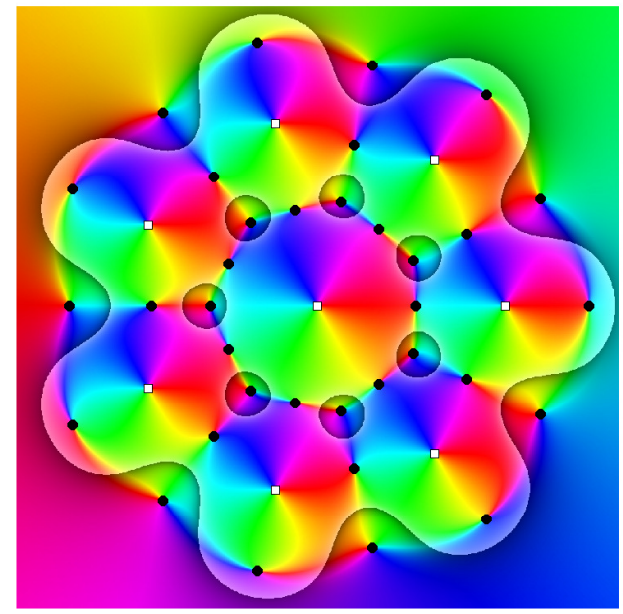

(b) Phase portrait of $R_{\varepsilon}(z)-\bar{z}$

Figure 1: Phase portraits of $R_{0}(z)-\bar{z}$ and $R_{\varepsilon}(z)-\bar{z}$. Black disks indicate zeros and white squares show poles.

same "zero creating effect" can be observed for a purely additive perturbation with $\frac{1}{z}$, resulting in the function $R_{0}(z)+\frac{\varepsilon}{z}-\bar{z}$. The analyses of this effect draw heavily from the rotational symmetry of $R_{0}(z)$. Hence it is tempting to regard this effect as a special property of $R_{0}(z)$.

The main goal of this paper is to prove, however, that the effect is generic to any rational harmonic function $R(z)-\bar{z}$, provided that it satisfies certain conditions at the perturbation point. In more details, we show that if $z_{0} \in \mathbb{C}$ is a zero of $R(z)-\bar{z}$ and if $n-1$ is the order of the first non-vanishing derivative of $R(z)$ at $z_{0}$, then $R(z)-\bar{z}$ can be perturbed by a pole at $z_{0}$ such that the perturbed rational harmonic function has (at least) $2 n$ additional zeros in a neighborhood of $z_{0}$, while the other zeros of $R(z)-\bar{z}$ "survive" the perturbation. The precise statement of this result is given in Theorem 3.1 below. In order to fully characterize this zero creating effect, perturbations with poles at arbitrary points $z_{0} \in \mathbb{C}$ are studied in Theorem 3.14 .

Adding poles to rational harmonic functions of the form $R(z)-\bar{z}$ in order to create new zeros has been used, for example, in [2, 4]. However, a complete mathematical characterization of this effect as in this work has not been given before.

We would also like to point out that the conceptually similar problem of constructing extremal harmonic polynomials $p(z)-\bar{z}=0$ appears to be more challenging. A class of examples that realizes the maximal number of zeros $3 \operatorname{deg}(p)-2$ (see [13]) is given by Geyer [8]. Recent progress concerning Wilmshurst's conjecture [24] on the maximal number of zeros for general harmonic polynomials $p(z)-\overline{q(z)}$ has been reported in [14].

The paper is organized as follows. In Section 2 we review tools from 
harmonic function theory that we need in our proofs. The major part of Section 3 consists of the proof of Theorem 3.1. We first show that sufficiently many zeros are created in a neighborhood of the perturbation point (Section 3.1), and we then show that the effect of the perturbation is local in the sense that the remaining zeros "survive" the perturbation (Section 3.2. In Section 4 we give several numerical illustrations. In Section 5 we state conclusions and some open questions in the context of our result.

\section{Mathematical background}

In this section we review the required mathematical background including the winding of continuous functions, the Poincare index of exceptional points and an argument principle that will allow to "count" zeros of $R(z)-\bar{z}$ in Section 3 .

The functions of the form $f(z)=R(z)-\bar{z}$ we consider in this paper are obviously not analytic. They are harmonic because for $z=x+i y$ they are twice continuously differentiable with respect to the real variables $x$ and $y$, and additionally $\frac{\partial^{2} f}{\partial x^{2}}+\frac{\partial^{2} f}{\partial y^{2}}=0$. In contrast to [6] we make no assumption whether a harmonic function is (locally) bijective or not. Since $f$ is harmonic, it has locally a representation of the form $f=h+\bar{g}$, where $h$ and $g$ are analytic functions. Both $h$ and $g$ are unique up to additive constants; see [7].

Roughly speaking, a harmonic function $h+\bar{g}$ is called sense-preserving if the analytic part $h$ is dominant, and sense-reversing if the co-analytic part $\bar{g}$ dominates. Since the exact definition simplifies for the harmonic functions of our interest, $f(z)=R(z)-\bar{z}$, we give the definition for this case only and refer to [7] and [21] for the general case.

Definition 2.1. Let $f(z)=R(z)-\bar{z}$ and $z_{0} \in \mathbb{C}$.

1. If $\left|R^{\prime}\left(z_{0}\right)\right|>1$, then $f(z)$ is called sense-preserving at $z_{0}$.

2. If $\left|R^{\prime}\left(z_{0}\right)\right|<1$, then $f(z)$ is called sense-reversing at $z_{0}$.

3. If $\left|R^{\prime}\left(z_{0}\right)\right|=1$, then $z_{0}$ is called a singular point of $f(z)$.

In either case, if additionally $f\left(z_{0}\right)=0$, then $z_{0}$ is respectively called a sense-preserving, sense-reversing or singular zero of $f(z)$. A zero $z_{0}$ of $f(z)$ that is not singular is called regular. If all zeros of $f(z)$ are regular, then the functions $f(z)$ and $R(z)$ are called regular.

We now turn our attention to the zeros of harmonic functions and their "multiplicity". It is clear that zeros of harmonic functions can, in general, not be "factored out". For example, each $z_{0} \in \mathbb{R}$ is a zero of the harmonic function $z-\bar{z}$, but there exists no harmonic function $g(z)$ with $z-\bar{z}=\left(z-z_{0}\right) g(z)$ or $z-\bar{z}=\overline{\left(z-z_{0}\right)} g(z)$. Even if all the zeros are isolated, as it is the case for harmonic polynomials of the form $p(z)-\bar{z}$ with $\operatorname{deg}(p)>1$, such decompositions typically do not exist, as the number of zeros may exceed 
the degree of the polynomial [8, 13]. In order to still "count" zeros, we will use the concept of the Poincaré index, which will be defined below; see Definition 2.4.

In order to define the Poincaré index, we need the following definition of the winding of a continuous function [1]; see also [22, p. 101] and [20, p. 29] (where the winding is called "degree"). Let $\Gamma$ be a rectifiable curve with parametrization $\gamma:[a, b] \rightarrow \Gamma$. Let $f: \Gamma \rightarrow \mathbb{C}$ be a continuous function with no zeros on $\Gamma$. Let $\Theta(z)$ denote a continuous branch of $\arg f(z)$ on $\Gamma$. The winding (or rotation) of $f(z)$ on the curve $\Gamma$ is defined as

$$
V(f ; \Gamma):=\frac{1}{2 \pi}(\Theta(\gamma(b))-\Theta(\gamma(a)))=\frac{1}{2 \pi} \Delta_{\Gamma} \arg f(z) .
$$

The winding is independent of the choice of the branch of $\arg f(z)$. The next proposition collects elementary properties of the winding.

Proposition 2.2 (see [1, p. 37] or [20, p. 29]). Let $\Gamma$ be a rectifiable curve, and let $f(z)$ and $g(z)$ be continuous and nonzero functions on $\Gamma$.

1. If $\Gamma$ is a closed curve, then $V(f ; \Gamma)$ is an integer.

2. If $\Gamma$ is a closed curve and if there exists a continuous and single-valued branch of the argument on $f(\Gamma)$, then $V(f ; \Gamma)=0$.

3. We have $V(f g ; \Gamma)=V(f ; \Gamma)+V(g ; \Gamma)$.

4. If $f(z)=c \neq 0$ is constant on $\Gamma$, then $V(f ; \Gamma)=0$.

The next result is a version of Rouché's theorem that is somewhat stronger than the classical one; see [1, p. 37]. Its formulation for analytic functions is due to Glicksberg [9]; see also [5]. We give a short proof for our more general setting.

Theorem 2.3 (Rouché's theorem). Let $\Gamma$ be a rectifiable closed curve, and let $f(z)$ and $g(z)$ be two continuous functions on $\Gamma$. If

$$
|f(z)+g(z)|<|f(z)|+|g(z)|, \quad z \in \Gamma,
$$

then $f(z)$ and $g(z)$ have the same winding on $\Gamma$, i.e. $V(f ; \Gamma)=V(g ; \Gamma)$.

Proof. Note first that (2) implies that $f(z)$ and $g(z)$ are nonzero on $\Gamma$. For $z \in \Gamma$, the inequality (2) yields $\left|\frac{f(z)}{g(z)}+1\right|<\left|\frac{f(z)}{g(z)}\right|+1$, hence $\frac{f(z)}{g(z)} \in \mathbb{C} \backslash[0, \infty[$. Thus $V\left(\frac{f}{g} ; \Gamma\right)=0$ since there is a continuous single-valued argument function on $\mathbb{C} \backslash\left[0, \infty\left[\right.\right.$. Using Proposition 2.2 we find $V(f ; \Gamma)=V\left(g \frac{f}{g} ; \Gamma\right)=V(g ; \Gamma)+$ $V\left(\frac{f}{g} ; \Gamma\right)=V(g ; \Gamma)$. 
Definition 2.4. Let the function $f(z)$ be continuous and different from zero in a punctured neighborhood $D$ of the point $z_{0}$. If $f(z)$ is either zero, not continuous, or not defined at $z_{0}$, then the point $z_{0}$ is called an isolated exceptional point of $f(z)$. The Poincaré index of the (isolated) exceptional point $z_{0}$ of the function $f(z)$ is defined as

$$
\operatorname{ind}\left(z_{0} ; f\right):=V(f ; \gamma)=\frac{1}{2 \pi} \Delta_{\gamma} \arg f(z) \in \mathbb{Z},
$$

where $\gamma$ is an arbitrary closed Jordan curve in $D$ surrounding $z_{0}$.

The Poincaré index is independent of the choice of $\gamma$; see [1], where exceptional points are called critical points. In [20, p. 44] the Poincaré index is called the "multiplicity of $f$ at $z_{0}$ ". It is a generalization of the multiplicity of a zero and pole of a meromorphic function, as is shown in the next example.

Example 2.5. Let $f(z)$ be a meromorphic function. The only isolated exceptional points of $f(z)$ are its zeros and poles. Suppose $f(z)=\left(z-z_{0}\right)^{m} g(z)$ holds in some neighborhood of $z_{0}$, where $g(z)$ is analytic and nonzero in this neighborhood, and $m \in \mathbb{Z}$. Then, for a sufficiently small circle $\gamma$ around $z_{0}$ lying in this neighborhood, Proposition 2.2 yields

$\operatorname{ind}\left(z_{0} ; f\right)=V(f ; \gamma)=V\left(\left(z-z_{0}\right)^{m} g(z) ; \gamma\right)=m V\left(z-z_{0} ; \gamma\right)+V(g ; \gamma)=m$.

Thus, for a zero of $f(z)(m>0)$ the Poincare index is the multiplicity of the zero, and for a pole $(m<0)$ it is (minus) the order of the pole.

With the Poincaré index, an argument principle for continuous functions with a finite number of exceptional points can be proven; see [1, p. 39] for the version below, or [20, p. 44].

Theorem 2.6. Let $\Gamma$ be a closed Jordan curve. Let $f(z)$ be continuous on and interior to $\Gamma$, except possibly for finitely many exceptional points $z_{1}, z_{2}, \ldots, z_{k}$ in the interior of $\Gamma$. Then

$$
\frac{1}{2 \pi} \Delta_{\Gamma} \arg f(z)=V(f ; \Gamma)=\operatorname{ind}\left(z_{1} ; f\right)+\operatorname{ind}\left(z_{2} ; f\right)+\ldots+\operatorname{ind}\left(z_{k} ; f\right) .
$$

This "abstract argument principle" includes as special cases the argument principle for meromorphic functions (see Example 2.5), and the argument principles for harmonic functions [7] and for harmonic functions with poles [21, Theorems 2.2 and 2.3].

We end this section with a discussion of the exceptional points and their Poincaré indices of $f(z)=R(z)-\bar{z}$, where $\operatorname{deg}(R) \geq 2$. The exceptional points of $f(z)$ are its zeros and its poles. The function $f(z)$ has a pole of order $m$ at $z_{0}$, if $z_{0}$ is a pole of order $m$ of $R(z)$. The assumption $\operatorname{deg}(R) \geq 2$ implies that $f(z)$ has at most $5(\operatorname{deg}(R)-1)$ zeros (cf. the Introduction). Hence all exceptional points of $f(z)$ are isolated and thus have a Poincaré index. 
The Poincaré index of a regular zero of a general harmonic function can be read off the power series of its analytic and co-analytic parts. This characterization was implicitly obtained in [7]. Under certain conditions the Poincaré index of a pole can be determined in a similar way [21]. Using [7, p. 412], Lemma 2.2 and the argument principle in [21], we obtain the following characterization for rational harmonic functions of the form $f(z)=R(z)-\bar{z}$.

Proposition 2.7. Let $f(z)=R(z)-\bar{z}$ with $\operatorname{deg}(R) \geq 2$.

1. A sense-preserving zero of $f(z)$ has Poincaré index +1 and a sensereversing zero of $f(z)$ has Poincaré index -1 .

2. If $z_{0}$ is a pole of $R(z)$ of order $m$, then $f(z)$ is sense-preserving in a neighborhood of $z_{0}$ and has Poincaré index $-m$ at $z_{0}$.

\section{Creating zeros by adding poles}

In Theorem 3.1, the main result of this paper, we generalize Rhie's construction as outlined in the Introduction. In short, the effect of an additive perturbation with a pole at a point $z_{0}$ where $R(z)-\bar{z}$ has a sense-reversing zero at which some derivatives of $R(z)$ vanish, can be roughly summarized as follows: New zeros appear "close to $z_{0}$ ", and existing zeros of $R(z)-\bar{z}$ "away from $z_{0}$ " do not disappear.

We denote by $D(z, r)$ and $\bar{D}(z, r)$ the open and the closed disks around $z \in \mathbb{C}$ with radius $r>0$, respectively. The open and closed annuli of radii $r>0$ and $s>0$ around $z \in \mathbb{C}$ are denoted by $A(z, r, s)$ and $\bar{A}(z, r, s)$, respectively.

Theorem 3.1. Let $f(z)=R(z)-\bar{z}$ with $\operatorname{deg}(R) \geq 2$ satisfy $\lim _{z \rightarrow \infty} f(z)=$ $\infty$. Further, let $z_{0} \in \mathbb{C}$ and the integer $n \geq 3$ satisfy

$$
f\left(z_{0}\right)=0, \quad R^{\prime}\left(z_{0}\right)=\ldots=R^{(n-2)}\left(z_{0}\right)=0, \quad \text { and } \quad R^{(n-1)}\left(z_{0}\right) \neq 0,
$$

and set $\eta:=\left(\frac{n}{n-1}\right)^{\frac{1}{2}}$. Then, for sufficiently small $\varepsilon>0$, the disk $\bar{D}\left(z_{0}, \eta \sqrt{\varepsilon}\right)$ contains no further zero of $f(z)$ and the function

$$
F(z):=f(z)+\frac{\varepsilon}{z-z_{0}}
$$

satisfies the following:

(i) $F(z)$ has at least $n$ zeros in $A\left(z_{0}, \eta^{-1} \sqrt{\varepsilon}, \sqrt{\varepsilon}\right)$, at least $n$ zeros in $A\left(z_{0}, \sqrt{\varepsilon}, \eta \sqrt{\varepsilon}\right)$, and no zeros in $\bar{D}\left(z_{0}, \eta^{-1} \sqrt{\varepsilon}\right)$. If $F(z)$ is regular, then at least $n$ of its zeros in $A\left(z_{0}, \eta^{-1} \sqrt{\varepsilon}, \sqrt{\varepsilon}\right)$ are sense-preserving and at least $n$ of its zeros in $A\left(z_{0}, \sqrt{\varepsilon}, \eta \sqrt{\varepsilon}\right)$ are sense-reversing.

(ii) Denote the regular zeros of $f(z)$ outside $\bar{D}\left(z_{0}, \eta \sqrt{\varepsilon}\right)$ by $z_{1}, \ldots, z_{N}$. Then there exist mutually disjoint disks $\bar{D}\left(z_{k}, r\right)$ such that $F(z)$ has exactly one zero in each $D\left(z_{k}, r\right)$, and the index of this zero is $\operatorname{ind}\left(z_{k} ; f\right)$.

(iii) If $f(z)$ is regular, then $f(z)$ and $F(z)$ have the same number of zeros outside $\bar{D}\left(z_{0}, \eta \sqrt{\varepsilon}\right)$. 
The proof of this result will be given in two parts, spanning the Sections 3.1 and 3.2. First we will give several remarks on the technical conditions on $f(z)$ and $F(z)$ we impose in this theorem.

Remark 3.2. 1 . The condition that $\lim _{z \rightarrow \infty} f(z)=\infty$ is rather nonrestrictive. First note that if $f(z)$ has a limit for $z \rightarrow \infty$, this limit is $\infty$. Further, $f(z)$ has no limit for $z \rightarrow \infty$ only when $R(z)=a_{1} z+\frac{p(z)}{q(z)}$, with $\left|a_{1}\right|=1$ and $\operatorname{deg}(p) \leq \operatorname{deg}(q)$. For all other $R(z)$, the limit $\lim _{z \rightarrow \infty} f(z)$ exists.

2. As shown in [11, p. 1081], the set of regular rational functions is dense in the space of all rational functions with respect to the supremum norm on the Riemann sphere. Hence, restricting $f(z)$ to be regular in (iii) is a very mild condition.

3. However, the effects of a perturbation on a non-regular function $f(z)$ can be quite unpleasant: Zeros of $f(z)$ far away from $z_{0}$ can vanish or additional zeros may appear. This is why the regularity assumptions in (ii) and (iii) are necessary.

In particular, Theorem 3.1 can be used to explain the maximality of Rhie's construction for a gravitational point lens, which we briefly discussed in the introduction. This is the content of the following Corollary; see also Section 4.1 and Figure 1.

Corollary 3.3. Under the assumptions of Theorem 3.1, suppose that $f(z)=$ $R(z)-\bar{z}$ is regular and has $3 n+1$ zeros, and that $\operatorname{deg}(R)=n$. Then, for sufficiently small $\varepsilon>0$, the function $R(z)+\frac{\varepsilon}{z-z_{0}}$ is extremal.

Proof. By Theorem 3.1 the function $F(z)=R(z)+\frac{\varepsilon}{z-z_{0}}-\bar{z}$ with sufficiently small $\varepsilon>0$ has at least $2 n$ zeros inside $A\left(z_{0}, \eta^{-1} \sqrt{\varepsilon}, \eta \sqrt{\varepsilon}\right.$ ) (cf. item (i)) and exactly $3 n$ zeros outside $\bar{D}\left(z_{0}, \eta \sqrt{\varepsilon}\right.$ ) (cf. item (iii)). Since $F(z)$ may have at most $5 n$ zeros, it in fact has exactly $5 n$ zeros, so that $R(z)+\frac{\varepsilon}{z-z_{0}}$ is extremal.

In the following Section 3.1 we prove part (i) of Theorem 3.1. The proof of parts (ii) and (iii) are the content of Section 3.2. Finally, in Section 3.3 . we analyze the effect of a perturbation with a pole at points $z_{0}$ that do not satisfy the assumptions of Theorem 3.1 .

\subsection{Behavior near $z_{0}$ (Proof of (i) in Theorem 3.1)}

The idea of our proof is the following: We approximate $F(z)$ by a truncation of the Laurent series of the analytic part of $F(z)$, and we show that this approximation has $2 n$ zeros near $z_{0}$ that carry over to zeros of $F(z)$, provided $\varepsilon$ is sufficiently small. 
Let

$$
R(z)=\sum_{k=0}^{\infty} \frac{R^{(k)}\left(z_{0}\right)}{k !}\left(z-z_{0}\right)^{k}
$$

be the series expansion of $R(z)$ at $z_{0}$ (convergent in some suitable disk around $\left.z_{0}\right)$. For the moment, consider any $\varepsilon>0$. We will specify below when $\varepsilon$ is "sufficiently small". Using (3) we can write

$$
\begin{aligned}
F(z) & =R(z)+\frac{\varepsilon}{z-z_{0}}-\bar{z} \\
& =\frac{R^{(n-1)}\left(z_{0}\right)}{(n-1) !}\left(z-z_{0}\right)^{n-1}+\sum_{k=n}^{\infty} \frac{R^{(k)}\left(z_{0}\right)}{k !}\left(z-z_{0}\right)^{k}+\frac{\varepsilon}{z-z_{0}}-\overline{z-z_{0}} .
\end{aligned}
$$

We substitute $w:=z-z_{0}$, so that $z_{0}$ is mapped to 0 . To simplify notation, we write again $F(w)$ instead of introducing a new notation $\widetilde{F}(w):=F(z)$. In the following we assume that the same substitution has been applied to $R(z)$ and $f(z)$ in order to obtain $R(w)$ and $f(w)$. We set $c:=\frac{R^{(n-1)}(0)}{(n-1) !} \neq 0$ and hence obtain

$$
F(w)=c w^{n-1}+\sum_{k=n}^{\infty} \frac{R^{(k)}(0)}{k !} w^{k}+\frac{\varepsilon}{w}-\bar{w} .
$$

Consider the function

$$
G(w):=c w^{n-1}+\frac{\varepsilon}{w}-\bar{w},
$$

obtained from $F(w)$ by truncation of the series expansion of $R(w)$. The zeros of $G(w)$ are the solutions of the equation

$$
c w^{n}-|w|^{2}+\varepsilon=0 .
$$

In the following, we will assume without loss of generality that $c>0$. Indeed, the solutions of $(6)$ with general $c \neq 0$ have the form $e^{-i \frac{\arg (c)}{n}} w$, where $w$ solves $|c| w^{n}-|w|^{2}+\varepsilon=0$.

The next three lemmata characterize the zeros of $G(w)$. First, we will derive conditions on $\varepsilon$ such that $G(w)$ admits a maximal number of zeros (Lemma 3.4). Then, in Lemma 3.5, we derive bounds on the moduli of certain such zeros that will be needed later. The sense of these zeros is determined in Lemma 3.6 .

Lemma 3.4. Let $n \geq 3, c>0$ and $0<\varepsilon<\varepsilon_{*}:=\frac{n-2}{n}\left(\frac{2}{n c}\right)^{\frac{2}{n-2}}$. Then (6) has exactly $3 n$ solutions $\rho_{1} e^{i \frac{(2 k+1) \pi}{n}}, \rho_{2} e^{i \frac{2 k \pi}{n}}, \rho_{3} e^{i \frac{2 k \pi}{n}}, 1 \leq k \leq n$, where $\rho_{1}<\sqrt{\varepsilon}<\rho_{2}<\left(\frac{2}{n c}\right)^{\frac{1}{n-2}}<\rho_{3}$.

Proof. Write $w=\rho e^{i \varphi}$ with $\rho>0$ and $\varphi \in \mathbb{R}$. Equation (6) is equivalent to

$$
c \rho^{n} e^{i n \varphi}-\rho^{2}+\varepsilon=0, \quad \text { or } \quad e^{i n \varphi}=\frac{\rho^{2}-\varepsilon}{c \rho^{n}} .
$$


Thus $e^{i n \varphi}$ is real and we distinguish the two cases $e^{i n \varphi}= \pm 1$.

If $e^{i n \varphi}=-1$, then $\rho^{2}<\varepsilon, \varphi=\frac{(2 k+1) \pi}{n}$ for some $k \in \mathbb{Z}$, and equation (7) becomes $c \rho^{n}+\rho^{2}-\varepsilon=0$. By Descartes' rule of signs (see [10, p. 442]), this equation has exactly one positive root, say $\rho_{1}<\sqrt{\varepsilon}$.

If $e^{i n \varphi}=+1$, then $\rho^{2}>\varepsilon, \varphi=\frac{2 k \pi}{n}$ for some $k \in \mathbb{Z}$, and (7) yields

$$
f_{+}(\rho):=c \rho^{n}-\rho^{2}+\varepsilon=0 .
$$

By Descartes' rule of signs $f_{+}(\rho)$ has 0 or 2 positive roots, counting multiplicities. We derive a necessary and sufficient condition on $\varepsilon$ such that $f_{+}(\rho)$ has two (distinct) positive roots. Since $f_{+}^{\prime}(\rho)=n c \rho^{n-1}-2 \rho=\rho\left(n c \rho^{n-2}-2\right)$, the only positive critical point of $f_{+}(\rho)$ is $\rho=\left(\frac{2}{n c}\right)^{\frac{1}{n-2}}$. From $f_{+}(0)=\varepsilon>0$ and $\lim _{\rho \rightarrow \infty} f_{+}(\rho)=\infty$, we see that $f_{+}(\rho)$ has two distinct positive roots if and only if $f_{+}\left(\left(\frac{2}{n c}\right)^{\frac{1}{n-2}}\right)<0$, which is equivalent to $\varepsilon<\varepsilon_{*}$. Hence, $f_{+}(\rho)$ has two distinct positive roots $\sqrt{\varepsilon}<\rho_{2}<\left(\frac{2}{n c}\right)^{\frac{1}{n-2}}<\rho_{3}$ if and only if $\varepsilon<\varepsilon_{*}$.

Lemma 3.5. In the notation of Lemma 3.4, if

$$
0<\varepsilon<\min \left\{\varepsilon_{*},\left(\frac{1}{n c}\right)^{\frac{2}{n-2}}\left(\frac{n}{n-1}\right)^{\frac{n}{n-2}},\left(\frac{1}{c(n-1)}\right)^{\frac{2}{n-2}}\left(\frac{n-1}{n}\right)^{\frac{n}{n-2}}\right\},
$$

then $G(w)$ has $3 n$ zeros, and we have

$$
\eta^{-1} \sqrt{\varepsilon}<\rho_{1}<\sqrt{\varepsilon}<\rho_{2}<\eta \sqrt{\varepsilon}, \quad \text { where } \eta:=\left(\frac{n}{n-1}\right)^{\frac{1}{2}} .
$$

Proof. Recall that $\rho_{1}$ is the positive root of $f_{-}(\rho):=c \rho^{n}+\rho^{2}-\varepsilon$. Note that $f_{-}(\sqrt{\varepsilon})>0$. We then have $\left(\frac{n-1}{n}\right)^{\frac{1}{2}} \sqrt{\varepsilon}<\rho_{1}<\sqrt{\varepsilon}$, if

$$
f_{-}\left(\left(\frac{n-1}{n}\right)^{\frac{1}{2}} \sqrt{\varepsilon}\right)=c\left(\frac{n-1}{n}\right)^{\frac{n}{2}} \varepsilon^{\frac{n}{2}}+\frac{n-1}{n} \varepsilon-\varepsilon=c\left(\frac{n-1}{n}\right)^{\frac{n}{2}} \varepsilon^{\frac{n}{2}}-\frac{1}{n} \varepsilon<0,
$$

which holds if and only if $\varepsilon<\left(\frac{1}{n c}\right)^{\frac{2}{n-2}}\left(\frac{n}{n-1}\right)^{\frac{n}{n-2}}$. To derive the bound for $\rho_{2}$, the smaller positive root of $f_{+}(\rho):=c \rho^{n}-\rho^{2}+\varepsilon$, note first that $f_{+}(\sqrt{\varepsilon})=$ $c \sqrt{\varepsilon}^{n}>0$. We then have $\sqrt{\varepsilon}<\rho_{2}<\left(\frac{n}{n-1}\right)^{\frac{1}{2}} \sqrt{\varepsilon}$, if

$$
f_{+}\left(\left(\frac{n}{n-1}\right)^{\frac{1}{2}} \sqrt{\varepsilon}\right)=c\left(\frac{n}{n-1}\right)^{\frac{n}{2}} \varepsilon^{\frac{n}{2}}-\frac{n}{n-1} \varepsilon+\varepsilon=c\left(\frac{n}{n-1}\right)^{\frac{n}{2}} \varepsilon^{\frac{n}{2}}-\frac{1}{n-1} \varepsilon<0,
$$

which holds if and only if $\varepsilon<\left(\frac{1}{c(n-1)}\right)^{\frac{2}{n-2}}\left(\frac{n-1}{n}\right)^{\frac{n}{n-2}}$.

Lemma 3.6. Let $\varepsilon>0$ satisfy condition (8). Then $G(w)$ in (5) is sensepreserving at its zeros $\rho_{1} e^{i \frac{(2 k+1) \pi}{n}}$ and sense-reversing at its zeros $\rho_{2} e^{i \frac{2 k \pi}{n}}$, $1 \leq k \leq n$.

Proof. We verify directly Definition 2.1 for both types of zeros. For ease of notation we denote the analytic part of $G(w)$ by $R_{G}(w):=c w^{n-1}+\frac{\varepsilon}{w}$. We have

$$
R_{G}^{\prime}(w)=(n-1) c w^{n-2}-\frac{\varepsilon}{w^{2}}=\frac{1}{w^{2}}\left((n-1) c w^{n}-\varepsilon\right) .
$$


Any zero $w^{\prime}$ of $G(w)$ is a solution of (6), thus satisfying $c\left(w^{\prime}\right)^{n}=\left|w^{\prime}\right|^{2}-\varepsilon$, so that

$$
R_{G}^{\prime}\left(w^{\prime}\right)=\frac{1}{\left(w^{\prime}\right)^{2}}\left((n-1)\left|w^{\prime}\right|^{2}-(n-1) \varepsilon-\varepsilon\right)=\frac{1}{\left(w^{\prime}\right)^{2}}\left((n-1)\left|w^{\prime}\right|^{2}-n \varepsilon\right)
$$

and

$$
\left|R_{G}^{\prime}\left(w^{\prime}\right)\right|=\left|(n-1)-n \frac{\varepsilon}{\left|w^{\prime}\right|^{2}}\right| .
$$

Since $\varepsilon$ satisfies (8), we have $\rho_{1}^{2}<\varepsilon<\rho_{2}^{2}<\frac{n}{n-1} \varepsilon$, see Lemma 3.5, which implies

$$
(n-1)-n \frac{\varepsilon}{\rho_{1}^{2}}<(n-1)-n \frac{\varepsilon}{\rho_{2}^{2}}<(n-1)-n \frac{n-1}{n}=0 .
$$

Then

$$
\left|R_{G}^{\prime}\left(\rho_{1} e^{i \frac{(2 k+1) \pi}{n}}\right)\right|=n \frac{\varepsilon}{\rho_{1}^{2}}-(n-1)>n-(n-1)=1,
$$

which shows that $G(w)$ is sense-preserving at the zeros $\rho_{1} e^{i \frac{(2 k+1) \pi}{n}}$, and

$$
\left|R_{G}^{\prime}\left(\rho_{2} e^{i \frac{2 k \pi}{n}}\right)\right|=n \frac{\varepsilon}{\rho_{2}^{2}}-(n-1)<n-(n-1)=1,
$$

which shows that $G(w)$ is sense-reversing at the zeros $\rho_{2} e^{i \frac{2 k \pi}{n}}$.

The preceding lemma concludes the discussion of the zeros of $G(w)$. In the following main result of this section we prove (i) of Theorem 3.1. We will show that the zeros $\rho_{1} e^{i \frac{(2 k+1) \pi}{n}}$ and $\rho_{2} e^{i \frac{i k \pi}{n}}$ of $G(w)$ give rise to zeros of $F(w)$.

Theorem 3.7. Let $n \geq 3$ and $c>0$ and set $\eta:=\left(\frac{n}{n-1}\right)^{\frac{1}{2}}$. Then, for all sufficiently small $\varepsilon>0$, the function $F(w)$ in (4) has at least $n$ zeros in $A\left(0, \eta^{-1} \sqrt{\varepsilon}, \sqrt{\varepsilon}\right)$, at least $n$ zeros in $A(0, \sqrt{\varepsilon}, \eta \sqrt{\varepsilon})$, and no zeros in $\bar{D}\left(0, \eta^{-1} \sqrt{\varepsilon}\right)$. If $F(w)$ is regular, then at least $n$ of its zeros in $A\left(0, \eta^{-1} \sqrt{\varepsilon}, \sqrt{\varepsilon}\right)$ are sense-preserving and at least $n$ of its zeros in $A(0, \sqrt{\varepsilon}, \eta \sqrt{\varepsilon})$ are sensereversing.

Proof. Let $\varepsilon$ satisfy (8), so that in particular $G(w)$ in (5) has $3 n$ zeros. We will show that the $2 n$ zeros of $G(w)$ discussed in Lemma 3.6 give rise to $2 n$ zeros of $F(w)$.

Consider a sense-preserving zero $w_{+}=\rho_{1} e^{\frac{i(2 k+1) \pi}{n}}$ of $G(w)$. From Lemma 3.5 we have $\eta^{-1} \sqrt{\varepsilon}<\left|w_{+}\right|<\sqrt{\varepsilon}$. In particular, $w_{+}$is the only exceptional point of $G(w)$ in the annular sector defined by the two radii $\eta^{-1} \sqrt{\varepsilon}$ and $\sqrt{\varepsilon}$, and the two half-lines $\arg (w)=\frac{2 k \pi}{n}$ and $\arg (w)=\frac{(2 k+2) \pi}{n}$; see Figure 2(a). Let $\Gamma^{+}=\left[\Gamma_{1}^{+}, \Gamma_{2}^{+}, \Gamma_{3}^{+}, \Gamma_{4}^{+}\right]$be the boundary curve of this annular sector as indicated in Figure 2(a), Since $G(w)$ is sense-preserving at $w_{+}$(see Lemma 3.6), we know that $V\left(G ; \Gamma^{+}\right)=+1$ and we will show next that $V\left(F ; \Gamma^{+}\right)=+1$. 
In order to apply Rouché's theorem (Theorem 2.3), we will show that

$$
|F(w)-G(w)|<|F(w)|+|G(w)|, \quad w \in \Gamma^{+} .
$$

From (4) and (5), we see that inside a disk around $w=0$ contained in the domain of convergence of the series of $R(w)$ we have $|F(w)-G(w)| \leq M|w|^{n}$, for some $M>0$ independent of $\varepsilon$. Hence, for sufficiently small $\varepsilon>0, \Gamma^{+}$is inside this disk. Thus, it suffices to show that $|G(w)|>M|w|^{n}$ on the arcs that compose $\Gamma^{+}$(trivially, $\left.|F(w)|+|G(w)| \geq|G(w)|\right)$.

For $w \in \Gamma_{1}^{+}$we have $|w|=\sqrt{\varepsilon}$, so that for sufficiently small $\varepsilon>0$,

$$
|G(w)|=\left.\left.|w|^{-1}\left|c w^{n}+\varepsilon-\right| w\right|^{2}\left|=c \sqrt{\varepsilon}^{n-1}>M \sqrt{\varepsilon}^{n}=M\right| w\right|^{n} .
$$

For $w \in \Gamma_{2}^{+}$or $w \in \Gamma_{4}^{+}$we have $w^{n}=|w|^{n}$ and $\eta^{-1} \sqrt{\varepsilon} \leq|w| \leq \sqrt{\varepsilon}$. If $\varepsilon>0$ is sufficiently small, then

$$
\begin{aligned}
|G(w)| & =\left.\left.|w|^{-1}\left|c w^{n}+\varepsilon-\right| w\right|^{2}|=| w\right|^{-1}\left(c|w|^{n}+\varepsilon-|w|^{2}\right) \geq c|w|^{n-1} \\
& \geq c \eta^{-(n-1)} \sqrt{\varepsilon}^{n-1}>M \sqrt{\varepsilon}^{n} \geq M|w|^{n} .
\end{aligned}
$$

For $w \in \Gamma_{3}^{+}$we have $|w|=\eta^{-1} \sqrt{\varepsilon}$, so that for sufficiently small $\varepsilon>0$,

$$
\begin{aligned}
|G(w)| & =\left.\left.|w|^{-1}\left|c w^{n}+\varepsilon-\right| w\right|^{2}|=| w\right|^{-1}\left|c w^{n}+\left(1-\eta^{-2}\right) \varepsilon\right| \\
& \geq|w|^{-1}\left(\left(1-\eta^{-2}\right) \varepsilon-c|w|^{n}\right) \geq \frac{1-\eta^{-2}}{\eta^{-1}} \sqrt{\varepsilon}-c \eta^{-(n-1)} \sqrt{\varepsilon}^{n-1} \\
& >M \eta^{-n} \sqrt{\varepsilon}^{n}=M|w|^{n} .
\end{aligned}
$$

Hence for sufficiently small $\varepsilon>0$ we find that $(9)$ is satisfied on $\Gamma^{+}$and thus $V\left(F ; \Gamma^{+}\right)=1$, so $F(w)$ has at least one zero inside this sector (by the argument principle). If $F(w)$ is regular, this zero is sense-preserving. Since $G(w)$ has $n$ zeros of type $w_{+}, F(w)$ has at least $n$ such zeros in the annulus $A\left(0, \eta^{-1} \sqrt{\varepsilon}, \sqrt{\varepsilon}\right)$.

We can use exactly the same reasoning as above to show that the sensereversing zeros $\rho_{2} e^{i \frac{2 k \pi}{n}}$ of $G(w)$ give zeros of $F(w)$. From Lemma 3.4 and Lemma 3.5 we see that $G(w)$ has $n$ such zeros inside $A(0, \sqrt{\varepsilon}, \eta \sqrt{\varepsilon})$. Now, fix $w_{-}=\rho_{2} e^{i \frac{2 k \pi}{n}}$. Consider the boundary curve $\Gamma^{-}=\left[\Gamma_{1}^{-}, \Gamma_{2}^{-}, \Gamma_{3}^{-}, \Gamma_{4}^{-}\right]$ of the annular sector defined by the radii $\sqrt{\varepsilon}$ and $\eta \sqrt{\varepsilon}$ and the half-lines $\arg (w)=\frac{(2 k-1) \pi}{n}$ and $\arg (w)=\frac{(2 k+1) \pi}{n}$ (again, see Figure 2(a)). As before, we show that $|G(w)|>M|w|^{n}$ on $\Gamma^{-}$.

The arc $\Gamma_{3}^{-}$has been treated already in $(10)$. For $w \in \Gamma_{1}^{-}$we have $|w|=\eta \sqrt{\varepsilon}$, so that for sufficiently small $\varepsilon>0$,

$$
\begin{aligned}
|G(w)| & =\left.\left.|w|^{-1}\left|c w^{n}+\varepsilon-\right| w\right|^{2}|=| w\right|^{-1}\left|c w^{n}+\left(1-\eta^{2}\right) \varepsilon\right| \\
& \geq|w|^{-1}\left(\left(\eta^{2}-1\right) \varepsilon-c|w|^{n}\right) \geq \frac{\eta^{2}-1}{\eta} \sqrt{\varepsilon}-c \eta^{n-1} \sqrt{\varepsilon}^{n-1} \\
& >M \eta^{n} \sqrt{\varepsilon}^{n}=M|w|^{n} .
\end{aligned}
$$




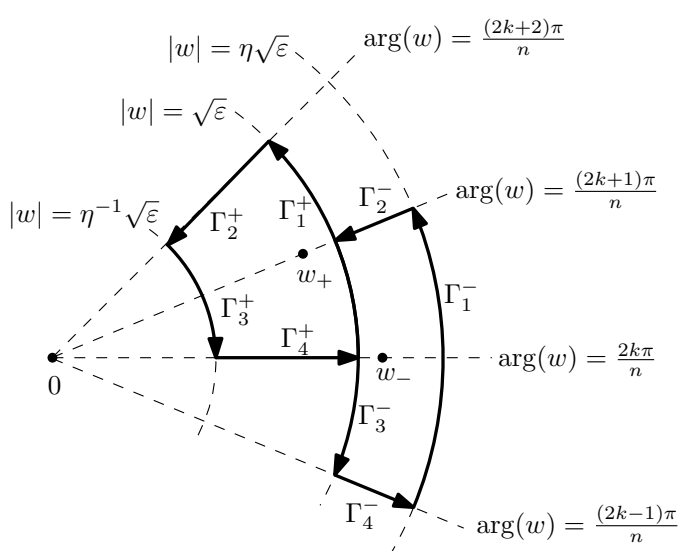

(a)

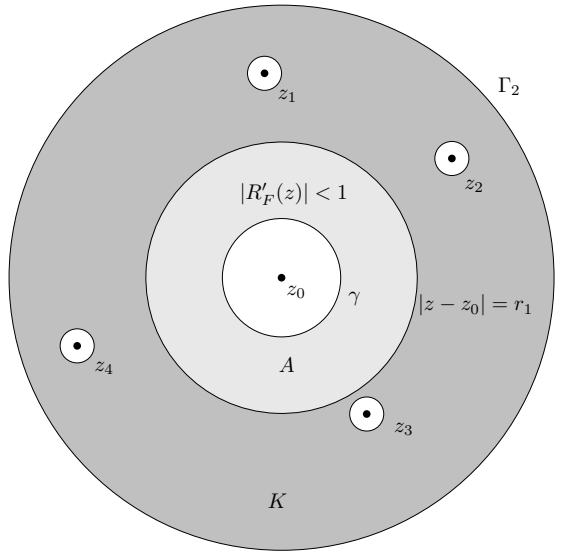

(b) $\gamma$ denotes the circle $\left|z-z_{0}\right|=\eta \sqrt{\varepsilon}$

Figure 2: Illustrations for the proofs of Theorems 3.7 (left) and 3.13 (right).

For $w \in \Gamma_{2}^{-}$or $w \in \Gamma_{4}^{-}$we have $w^{n}=-|w|^{n}$. Using that $\sqrt{\varepsilon} \leq|w| \leq \eta \sqrt{\varepsilon}$ we compute, for a sufficiently small $\varepsilon>0$,

$$
\begin{aligned}
|G(w)| & =\left.\left.\left.|w|^{-1}\left|c w^{n}+\varepsilon-\right| w\right|^{2}|=| w\right|^{-1}|-c| w\right|^{n}+\varepsilon-|w|^{2} \mid \\
& =|w|^{-1}\left(c|w|^{n}+|w|^{2}-\varepsilon\right) \geq c|w|^{n-1} \geq c \sqrt{\varepsilon}^{n-1}>M \eta^{n} \sqrt{\varepsilon}^{n} \geq M|w|^{n} .
\end{aligned}
$$

As before we can now conclude that $V\left(F ; \Gamma^{-}\right)=-1$, so $F(w)$ has at least one zero inside $\Gamma^{-}$, which is sense-reversing if $F(w)$ is regular. In total, $n$ such zeros exist.

In order to complete the proof, let $\gamma$ denote the circle $|w|=\eta^{-1} \sqrt{\varepsilon}$. From the computation (11) we see that Rouché's theorem applies to $F(w)$ and $G(w)$ on $\gamma$. Since $G(w)$ has a simple pole and no zeros inside $\gamma$, we obtain $V(F ; \gamma)=V(G ; \gamma)=-1$.

Let $R_{F}(w):=R(w)+\frac{\varepsilon}{w}$. For $0 \neq w \in \bar{D}\left(0, \eta^{-1} \sqrt{\varepsilon}\right)$ we compute

$$
\left|R_{F}^{\prime}(w)\right|=\left|R^{\prime}(w)-\frac{\varepsilon}{w^{2}}\right| \geq \frac{\varepsilon}{|w|^{2}}-\left|R^{\prime}(w)\right| \geq \eta^{2}-\left|R^{\prime}(w)\right|>1
$$

for sufficiently small $\varepsilon>0$, since $R^{\prime}(0)=0$. This shows that $F(w)$ is sensepreserving on the disk $\bar{D}\left(0, \eta^{-1} \sqrt{\varepsilon}\right)$. Since 0 is a simple pole of $F(w)$ with Poincaré index $-1=V(F ; \gamma)$, there are no zeros of $F(w)$ in this disk.

Remark 3.8. Theorem 3.7 gives a lower bound for $2 n$ additional zeros close to 0 . However, the proof does not show that there are exactly $2 n$ such zeros, because the sector enclosed by $\Gamma^{+}$may contain an open region where $\left|R_{F}^{\prime}(w)\right|<1$. In that case there may exist additional pairs of zeros inside this sector so that the total winding of +1 is maintained (similarly for $\Gamma^{-}$). We suspect that for sufficiently small $\varepsilon>0$ exactly $2 n$ additional zeros occur; but see also Section 4.2, where more than $2 n$ additional zeros occur for somewhat larger $\varepsilon$. 
We end this section with a result that follows easily from the proof of Theorem 3.7 and that will be helpful in the proof of Theorem 3.13 below.

Corollary 3.9. Under the assumptions of Theorem 3.7, we have $V(F ; \gamma)=$ -1 , where $\gamma$ is the circle $|w|=\eta \sqrt{\varepsilon}$.

Proof. From (12) we see that Rouché's theorem (Theorem 2.3) applies to $F(w)$ and $G(w)$ on the circle $\gamma$. But $G(w)$ has exactly one simple pole, $n$ sense-preserving and $n$ sense-reversing zeros inside $\gamma$, so $V(F ; \gamma)=V(G ; \gamma)=$ -1 .

\subsection{Behavior away from $z_{0}$ (Proof of (ii) and (iii) in Theo- rem 3.1)}

In the previous section we substituted $w=z-z_{0}$ because it simplified the notation. This is no longer necessary, so we work in the $z$ variable again. We will first prove (ii) of Theorem 3.1. but for a slightly more general setting, where we do not require $f\left(z_{0}\right)=0$. This setting will be needed in the proof of Theorem 3.14.

Theorem 3.10. Let $f(z)=R(z)-\bar{z}$ with $\operatorname{deg}(R) \geq 2$, and $z_{0} \in \mathbb{C}$. Let $z_{1}, \ldots, z_{N} \in \mathbb{C}$ be the regular zeros of $f(z)$, except, possibly, $z_{0}$. Then there exist mutually disjoint disks $\bar{D}\left(z_{k}, r\right)$ not containing $z_{0}$ with the following property: For all sufficiently small $\varepsilon>0$ the function

$$
F(z)=f(z)+\frac{\varepsilon}{z-z_{0}}
$$

has exactly one zero in $D\left(z_{k}, r\right)$, and the index of this zero is $\operatorname{ind}\left(z_{k} ; f\right)$.

Proof. Let $z_{1}, \ldots, z_{M}, M \geq N$, be all zeros of $f(z)$, except, possibly, $z_{0}$. Choose $r>0$ with the following properties:

1. The disks $\bar{D}\left(z_{k}, r\right)$ do not intersect $(k=1, \ldots, M)$ and do neither contain $z_{0}$ nor the poles of $R(z)$.

2. If $z_{k}$ is a regular zero, $f(z)$ is either sense-preserving or sense-reversing on $\bar{D}\left(z_{k}, r\right)$.

Fix a regular zero $z_{k}, 1 \leq k \leq N$. Let $\Gamma$ denote the circle around $z_{k}$ with radius $r$. By construction, the continuous function $z \mapsto\left|f(z)\left(z-z_{0}\right)\right|$ admits a positive minimum on the compact set $\Gamma$. For any

$$
0<\varepsilon<\frac{1}{2} \min _{z \in \Gamma}\left|f(z)\left(z-z_{0}\right)\right|
$$

we then have

$$
|F(z)|=\left|f(z)+\frac{\varepsilon}{z-z_{0}}\right| \geq|f(z)|-\left|\frac{\varepsilon}{z-z_{0}}\right|>\frac{\varepsilon}{\left|z-z_{0}\right|}, \quad z \in \Gamma,
$$

from which we find $|F(z)-f(z)|<|F(z)|+|f(z)|$ for $z \in \Gamma$. By Rouché's theorem (Theorem 2.3) we have $V(F ; \Gamma)=V(-f ; \Gamma)= \pm 1$, since $f(z)$ has 
exactly one regular zero in the interior of $\Gamma$. Thus $F(z)$ also has (at least) one zero in the interior of $\Gamma$ (by Theorem 2.6, see also the degree principle [20, Section 2.3.6] or [1, Theorem 2.3]).

Write $R_{F}(z)=R(z)+\frac{\varepsilon}{z-z_{0}}$, so that $F(z)=R_{F}(z)-\bar{z}$. Now suppose that $f(z)$ is sense-preserving at $z_{k}$, so that $\left|R^{\prime}(z)\right|>1$ on $\bar{D}\left(z_{k}, r\right)$. Note that $R_{F}^{\prime}(z)=R^{\prime}(z)-\frac{\varepsilon}{\left(z-z_{0}\right)^{2}}$, so that $\left|R_{F}^{\prime}(z)\right|>1$ on the disk if $\varepsilon$ is chosen sufficiently small. Therefore $F(z)$ is also sense-preserving and its zeros have positive index $(+1$, see Proposition 2.7). Then $V(F ; \Gamma)=V(f ; \Gamma)=$ $\operatorname{ind}\left(z_{k} ; f\right)=+1$ shows that $F(z)$ has exactly one zero in this disk. A similar reasoning holds for sense-reversing zeros of $f(z)$.

Remark 3.11. If all zeros of $f(z)$ have merely nonzero index, the first part of the proof still shows that $F(z)$ has at least one zero near every zero $z_{k} \neq z_{0}$ of $f(z)$.

Lemma 3.12. Let $f(z)=R(z)-\bar{z}$ with $\operatorname{deg}(R) \geq 2$. Let $z_{0}, \zeta \in \mathbb{C}$ with $z_{0} \neq \zeta$ and $f(\zeta) \neq 0$. Then there exists a neighborhood of $\zeta$ in which, for all sufficiently small $\varepsilon>0$, the function

$$
F(z)=f(z)+\frac{\varepsilon}{z-z_{0}}
$$

has no zeros.

Proof. Let us assume that $\zeta$ is not a pole of $R(z)$, else there is nothing to show. Then there exists $0<r<\left|z_{0}-\zeta\right|$ such that $f(z)$ is continuous and nonzero on $\bar{D}(\zeta, r)$. For

$$
\varepsilon<\min _{z \in \bar{D}(\zeta, r)}\left|\left(z-z_{0}\right) f(z)\right|
$$

(the right hand side is positive by the choice of $r$ ), we see from

$$
|F(z)| \geq|f(z)|-\frac{\varepsilon}{\left|z-z_{0}\right|}
$$

that $F(z)$ has no zeros in $\bar{D}(\zeta, r)$.

The following theorem completes the discussion of points "away" from the perturbation point $z_{0}$. Together with Theorem 3.7 and Theorem 3.10 , it implies Theorem 3.1 .

Theorem 3.13. In the notation and under the assumptions of Theorem 3.1, let additionally $f(z)$ be regular. Then for sufficiently small $\varepsilon>0$, the functions $F(z)$ and $f(z)$ have the same number of zeros outside $\bar{D}\left(z_{0}, \eta \sqrt{\varepsilon}\right)$. More precisely:

1. If $z_{1}, \ldots, z_{N}$ are the zeros of $f(z)$ with $\left|z_{k}-z_{0}\right|>\eta \sqrt{\varepsilon}$, then there exist mutually disjoint disks $\bar{D}\left(z_{k}, r\right), 1 \leq k \leq N$, such that $F(z)$ has exactly one zero in each $D\left(z_{k}, r\right)$, and the index of this zero is $\operatorname{ind}\left(z_{k} ; f\right)$. 


\section{2. $F(z)$ has no further zeros outside $\bar{D}\left(z_{0}, \eta \sqrt{\varepsilon}\right)$.}

Proof. Since $\lim _{z \rightarrow \infty} f(z)=\infty$, there exists $r_{2}>0$ such that $|f(z)| \geq 1$ for $\left|z-z_{0}\right| \geq r_{2}$. Further we can choose $r_{2}$ so that $\Gamma_{2}=\left\{z:\left|z-z_{0}\right|=r_{2}\right\}$ contains all poles of $f(z)$ in its interior. For $\left|z-z_{0}\right| \geq r_{2}$ we have

$$
|F(z)|=\left|f(z)+\frac{\varepsilon}{z-z_{0}}\right| \geq|f(z)|-\frac{\varepsilon}{\left|z-z_{0}\right|} \geq 1-\frac{\varepsilon}{r_{2}},
$$

which is positive for sufficiently small $\varepsilon>0$. Thus for each such $\varepsilon$ the function $F(z)$ has no zeros on or exterior to $\Gamma_{2}$. We further have

$$
|F(z)-f(z)|=\frac{\varepsilon}{\left|z-z_{0}\right|}=\frac{\varepsilon}{r_{2}}<1 \leq|f(z)| \leq|F(z)|+|f(z)|, \quad z \in \Gamma_{2} .
$$

Hence Rouché's theorem (Theorem 2.3 implies $V\left(F ; \Gamma_{2}\right)=V\left(f ; \Gamma_{2}\right)$. By Theorem 3.10 each zero of $f(z)$ inside $\Gamma_{2}$ has a corresponding zero of $F(z)$ with same index (except for $z_{0}$ ). We will now show that if $\varepsilon>0$ is sufficiently small, then $F(z)$ has no further zeros inside $\Gamma_{2}$ than implied by Theorem 3.7 and Theorem 3.10. In order to achieve this, we will use Lemma 3.12 combined with a compactness argument.

By assumption $R^{\prime}\left(z_{0}\right)=0$. Thus we can choose $r_{1}>0$ such that $\left|R^{\prime}(z)\right|<\frac{1}{n}$ for $\left|z-z_{0}\right| \leq r_{1}$, and such that $z_{0}$ is the only zero of $f(z)$ in $\left|z-z_{0}\right| \leq r_{1}$. We define the compact set $K$ as follows. Consider the closed annulus $r_{1} \leq\left|z-z_{0}\right| \leq r_{2}$ and denote by $z_{1}, \ldots, z_{N}$ the zeros of $f(z)$ in that annulus. By Theorem 3.10, there are mutually disjoint $\bar{D}\left(z_{k}, r\right)$, $0 \leq k \leq N$, such that $F(z)$ has exactly one zero $z_{k}^{\prime} \in D\left(z_{k}, r\right)$ of the same index, $1 \leq k \leq N$. Cutting out these neighborhoods in the annulus, we obtain the compact set $K$ (Figure 2(b)) .

For each $\zeta \in K$ we have $f(\zeta) \neq 0$, so there exists a neighborhood of $\zeta$ as in Lemma 3.12. These neighborhoods constitute an open covering of $K$, of which a finite subset is sufficient to cover $K$. On each neighborhood $F(z)$ is nonzero for all sufficiently small $\varepsilon$; see Lemma 3.12. Hence, only a finite number of smallness constraints on $\varepsilon$ are sufficient to guarantee that $F(z)$ has no zeros inside $K$.

Recall that inside each of the cut out disks $D\left(z_{k}, r\right), 1 \leq k \leq N$, the function $F(z)$ has exactly one zero of same index as $f(z)$. Thus, it remains to show that $F(z)$ has no additional zeros inside the annulus $A:=$ $A\left(z_{0}, \eta \sqrt{\varepsilon}, r_{1}\right)$. As before set $R_{F}(z)=R(z)+\frac{\varepsilon}{z-z_{0}}$. Then, for $z \in A$,

$$
\left|R_{F}^{\prime}(z)\right| \leq\left|R^{\prime}(z)\right|+\frac{\varepsilon}{\left|z-z_{0}\right|^{2}}<\frac{1}{n}+\frac{n-1}{n}=1,
$$

and thus $F(z)$ is sense-reversing on $A$. Denote by $\gamma$ the circle $\left\{z:\left|z-z_{0}\right|=\right.$ $\eta \sqrt{\varepsilon}$, and by $n_{-}$the number of (sense-reversing) zeros of $F(z)$ in $A$. By the argument principle we find

$$
-1+\sum_{k=1}^{N} \operatorname{ind}\left(z_{k} ; f\right)=V\left(f ; \Gamma_{2}\right)=V\left(F ; \Gamma_{2}\right)=V(F ; \gamma)+\sum_{k=1}^{N} \operatorname{ind}\left(z_{k}^{\prime} ; F\right)-n_{-},
$$


which shows $n_{-}=0$, since $\operatorname{ind}\left(z_{k} ; f\right)=\operatorname{ind}\left(z_{k}^{\prime} ; F\right)$ for $k=1, \ldots, N$, and $V(F ; \gamma)=-1 ;$ see Corollary 3.9 .

\subsection{Perturbation at arbitrary points}

We now consider perturbations at points where the assumptions of Theorem 3.1 are not satisfied. The situation is simpler than in the setting of Theorem 3.1 and for the proof the same techniques as in Section 3.1 can be applied. We therefore only give a sketch of the proof. Furthermore, we assume for simplicity that both $f(z)$ and $F(z)$ are regular, although this requirement could be weakened somewhat.

Theorem 3.14. Let $f(z)=R(z)-\bar{z}$ with $\operatorname{deg}(R) \geq 2$ be regular and satisfy $\lim _{z \rightarrow \infty} f(z)=\infty$, and let $z_{0} \in \mathbb{C}$. For sufficiently small $\varepsilon>0$, if

$$
F(z)=f(z)+\frac{\varepsilon}{z-z_{0}}
$$

is regular, then the following holds:

1. If $z_{0}$ is a pole of $f(z)$, then $F(z)$ and $f(z)$ have the same number of zeros.

2. If $0<\left|f\left(z_{0}\right)\right|<\infty$, then there exists $r>0$ such that $0<|f(z)|<\infty$ on $D\left(z_{0}, r\right)$, and $F(z)$ has at least one sense-preserving zero in $D\left(z_{0}, r\right)$.

3. If $f\left(z_{0}\right)=0$, and $\left|R^{\prime}\left(z_{0}\right)\right|>1$, there exists $r>0$ such that $F(z)$ has at least two sense-preserving zeros in $D\left(z_{0}, r\right)$.

4. If $f\left(z_{0}\right)=0$, and $0<\left|R^{\prime}\left(z_{0}\right)\right|<1$, there exists $r>0$ such that $F(z)$ has at least two sense-preserving zeros and two sense-reversing zeros in $D\left(z_{0}, r\right)$.

Further, in 2, 3. and 4, $F(z)$ and $f(z)$ have the same number of zeros outside $D\left(z_{0}, r\right)$.

Proof. (Sketch)

In 1. we have $R(z)=\left(z-z_{0}\right)^{-m} \widetilde{R}(z)$ with $\widetilde{R}\left(z_{0}\right) \notin\{0, \infty\}, m \geq 1$. In some disk $\bar{D}\left(z_{0}, \delta\right)$ we have $|z| \leq M$ and $|\widetilde{R}(z)| \geq M^{\prime}>0$. If need be, reduce $\delta$ such that $\delta^{m}<\frac{M^{\prime}}{2 M}$ holds. Noting that

$$
R_{F}(z)=R(z)+\frac{\varepsilon}{z-z_{0}}=\frac{1}{\left(z-z_{0}\right)^{m}}\left(\widetilde{R}(z)+\varepsilon\left(z-z_{0}\right)^{m-1}\right),
$$

one can compute that $|F(z)|>0$ on $\bar{D}\left(z_{0}, \delta\right)$ for all sufficiently small $\varepsilon$. Consider the annulus $A\left(z_{0}, \delta, r_{2}\right)$ which contains all zeros of $f(z)\left(r_{2}\right.$ is chosen as in the proof of Theorem 3.13). After cutting out appropriate disks about the zeros of $f(z)$, a compact set $K$ remains. As in the proof of Theorem 3.13 . for sufficiently small $\varepsilon>0$, each disk around a zero of $f(z)$ contains exactly one zero of $F(z)$, and the set $K$ does not contain any zeros of $F(z)$.

In 2. there exists $r>0$ such that $0<|f(z)|<\infty$ on $\bar{D}\left(z_{0}, r\right)$. By the argument principle the winding of $f(z)$ along the boundary curve is 0 . Apply Rouché's theorem (Theorem 2.3) to $f(z)$ and $F(z)$ on the boundary to see 
that $F(z)$ has at least one sense-preserving zero inside this disk, provided $\varepsilon>0$ is sufficiently small.

Let $r_{2}$ and $\Gamma_{2}$ be defined as in the proof of Theorem 3.13, and construct the compact set $K$ by removing from the annulus $\bar{A}\left(z_{0}, r, r_{2}\right)$ suitable disks centered at the zeros of $f(z)$. Proceeding exactly as in the proof of Theorem 3.13 we see that $F(z)$ and $f(z)$ have the same number of zeros outside $D\left(z_{0}, r\right)$.

In both 3. and 4. we substitute $w:=z-z_{0}$ for simplicity of notation, as in Section 3.1. Set $c:=R^{\prime}(0)$, which can be assumed to be real positive. Truncation of the Laurent series of the analytic part of $F(w)=f(w)+\frac{\varepsilon}{w}$ yields the function $G(w)=c w+\frac{\varepsilon}{w}-\bar{w}$.

In 3. we have $c>1$ and one computes that $G(w)$ has exactly two sensepreserving zeros $\pm i\left(\frac{\varepsilon}{1+c}\right)^{\frac{1}{2}}$. Applying Rouché's theorem to $F(w)$ and $G(w)$ on the circle $|w|=\sqrt{\varepsilon}$ gives the result. An example is shown in Section 4.3 .

Since $c>1$, there exists $r_{1}>0$ such that $\left|R^{\prime}(w)\right|>\frac{1+c}{2}$ on $\bar{D}\left(0, r_{1}\right)$. We then have for $w \in A:=A\left(0, \sqrt{\varepsilon}, r_{1}\right)$

$$
\left|R_{F}^{\prime}(w)\right|=\left|R^{\prime}(w)-\frac{\varepsilon}{w^{2}}\right| \geq\left|R^{\prime}(w)\right|-\frac{\varepsilon}{|w|^{2}} \geq 1+\frac{c-1}{2}-\frac{\varepsilon}{r_{1}^{2}},
$$

which is larger than 1 if $\varepsilon$ is chosen sufficiently small. Thus $F(w)$ is sensepreserving on $A$. Now, the same reasoning as in the proof of Theorem 3.13 (with the modified $A$ ) shows that $F(w)$ and $f(w)$ have the same number of zeros outside $D\left(z_{0}, r\right)$, where $r=\sqrt{\varepsilon}$.

In 4. we have $0<c<1$ and one computes that $G(w)$ has exactly the two sense-preserving zeros $\pm i\left(\frac{\varepsilon}{1+c}\right)^{\frac{1}{2}}$, and the two sense-reversing zeros $\pm\left(\frac{\varepsilon}{1-c}\right)^{\frac{1}{2}}$. Applying Rouché's theorem to $F(w)$ and $G(w)$ on $\gamma_{1}=\{w \in \mathbb{C}:|w|=\sqrt{\varepsilon}\}$ shows that $F(w)$ has two sense-preserving zeros inside $\gamma_{1}$, and applying it on the circle $\gamma_{2}=\left\{w \in \mathbb{C}:|w|=\frac{2}{\sqrt{1-c}} \sqrt{\varepsilon}\right\}$ shows that $F(w)$ has two sensereversing zeros between $\gamma_{1}$ and $\gamma_{2}$. An example for this case can be seen in Section 4.4, more specifically in Figure $5(\mathrm{~b})$.

Since $0<c<1$ there exists $r_{1}>0$ such that $\left|R^{\prime}(w)\right|<\frac{1+c}{2}$ on $\bar{D}\left(0, r_{1}\right)$. Then, we have for $w \in A:=A\left(0, \frac{2}{\sqrt{1-c}} \sqrt{\varepsilon}, r_{1}\right)$

$$
\left|R_{F}^{\prime}(w)\right|=\left|R^{\prime}(w)-\frac{\varepsilon}{w^{2}}\right| \leq\left|R^{\prime}(w)\right|+\frac{\varepsilon}{|w|^{2}} \leq \frac{1+c}{2}+\varepsilon \frac{1-c}{4 \varepsilon}<1,
$$

so that $F(w)$ is sense-reversing in $A$. The same reasoning as in the proof of Theorem 3.13 now shows that $F(w)$ and $f(w)$ have the same number of zeros outside $D\left(z_{0}, r\right)$, where $r=\frac{2}{\sqrt{1-c}} \sqrt{\varepsilon}$.

\section{Examples}

We briefly discuss the Poincaré index and its connection with phase portraits. (Recall the example given in the Introduction; see Figure 1.) Let $z_{0}$ be an isolated exceptional point of the continuous function $f(z)$, and let $\gamma$ be a 
circle around $z_{0}$ suitable for the computation of $\operatorname{ind}\left(z_{0} ; f\right)$. Clearly, the Poincaré index measures the overall change of the argument of $f(z)$ while $z$ travels once around $\gamma$ (in the positive sense). This corresponds exactly to the chromatic number of $\gamma$, as discussed in [23, p. 772]. Thus, less formally, the Poincaré index corresponds to the number of times each color appears in the phase portrait while travelling once around $z_{0}$, and the sign of the Poincare index is revealed by the ordering in which the colors appear. This observation allows to determine the Poincare index of an isolated exceptional point of $f(z)$ by looking at the phase portrait of $f(z)$.

For the color scheme we use in the phase portraits, the color ordering while travelling around some point is exemplified for the indices $+1,-1$ and -2 as follows (left to right):
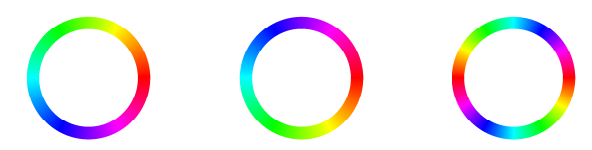

This is the same "color wheel" as used, for example, in [23]. For the computation of the phase portraits in this paper we used a slightly modified version of the MATLAB package "Phase Plots of Complex Functions" by Elias Wegert 1 .

Thus the index of an exceptional point of $f(z)=R(z)-\bar{z}$ can be determined from the phase portrait. Poles and zeros of $f(z)$, which are the only exceptional points, are marked with white squares (poles) or black disks (zeros). Additionally, all phase portraits are visually divided in slightly brightened regions, where $f(z)$ is sense-preserving (i.e., the analytic part dominates), and slightly darkened regions, where $f(z)$ is sense-reversing (i.e., the co-analytic part dominates). Notice that the indices of zeros in the latter case are always -1 , while the indices of sense-preserving zeros are +1 .

\subsection{Circular point lenses}

Recall Rhie's construction described in the Introduction, which is based on the function $R_{0}(z)=\frac{z^{d-1}}{z^{d}-r^{d}}$, for some $d \geq 2$ and $r>0$. It is easy to see that $R_{0}^{(k)}(0)=0$ for $1 \leq k \leq d-2$, while $R_{0}^{(d-1)}(0) \neq 0$. An application of (i) in Theorem 3.1 with $n=d$ shows that $F(z)=R_{0}(z)+\frac{\varepsilon}{z}-\bar{z}$ has $2 d$ zeros located in two annuli around the perturbation point $z_{0}=0$. Moreover, if $r>0$ and $\varepsilon>0$ are sufficiently small, then there exist further $3 d$ zeros corresponding to the $3 d$ zeros of $R_{0}(z)-\bar{z}$ away from $z_{0}=0$. The results in [16] show that the same perturbation behavior can be observed for Rhie's original function $(1-\varepsilon) R_{0}(z)+\frac{\varepsilon}{z}-\bar{z}$. A numerical example for this function with $d=7$ is shown in Figure 1 .

\footnotetext{
${ }^{1}$ http://www.visual. wegert.com/
} 


\subsection{Generation of more than $2 n$ zeros}

Theorem 3.7 ( $i$ ) gives the lower bound $2 n$ on the number of additional zeros due to the perturbation. While we suspect that actually exactly $2 n$ zeros appear for sufficiently small $\varepsilon$ (see also Remark 3.8), we will now see that more than $2 n$ zeros may appear if $\varepsilon$ is not small enough.

Consider the function

$$
R(z)=\frac{\frac{1}{6} z^{3}+\frac{1}{20} z^{2}}{z^{4}-\frac{1}{10}},
$$

for which $R(0)=R^{\prime}(0)=0,\left|R^{\prime \prime}(0)\right|=1$ and $\left|R^{\prime \prime \prime}(0)\right|=10$. A phase portrait of $R(z)-\bar{z}$ is shown in Figure 3(a),

Figure 3(b) shows a phase portrait of $R(z)+\frac{\varepsilon}{z}-\bar{z}$ with $\varepsilon=0.1$. The perturbation results in 8 additional zeros in the vicinity of 0 , although $n=3$. In this example the first non-vanishing derivative is dominated by a higher derivative and hence our analysis from Section 3 does not apply, since $\varepsilon$ is not small enough.

However, the second derivative becomes dominant as soon as $\varepsilon$ is sufficiently small. Figure 3(c) shows the result of perturbing with $\varepsilon=0.05$. Now only $2 n=6$ additional zeros occur, as suggested by Theorem 3.1. If $\varepsilon$ is reduced further, as can be seen in Figure 3(d) for $\varepsilon=0.01$, the additional zeros approach those of the function $G(w)$ in Section 3 , but the number of additional zeros (six) remains the same.

\subsection{Perturbing a sense-preserving zero}

Figure 4 shows the result of perturbing a sense-preserving zero by a pole. The left plot shows a randomly generated ${ }^{2}$ rational harmonic function $f(z)=$ $R(z)-\bar{z}$, where $R(z)$ is of degree two (both the nominator and denominator polynomials of $R(z)$ have degree two) and extremal. The point $z_{0}$ is a sensepreserving zero of $f(z)$ with $\left|R^{\prime}\left(z_{0}\right)\right| \approx 1.176>1$. We add a pole at $z_{0}$ with $\varepsilon=0.025$; the resulting function is shown in Figure 4 (right). As suggested by Theorem 3.14, two sense-preserving zeros appear.

\subsection{Iterative perturbation}

Starting from the randomly chosen extremal rational function $R(z)$ of degree two introduced in the previous section, our goal is now to successively add two poles at sense-reversing zeros such that extremality is maintained by each perturbation. We will thus obtain extremal rational functions of degrees three and four. This procedure is displayed in Figure 5, which we will discuss in detail now.

\footnotetext{
${ }^{2}$ More precisely, we chose 5 points in the complex plane, with real and imaginary parts drawn uniformly at random from $[-1,1]$. We then constructed $R(z)$ such that these random points are the zeros of $R(z)-\bar{z}$.
} 
The initial situation with $R_{1}(z)=R(z)$ is depicted in Figure $5(\mathrm{a})$, where we plot the phase portrait of $R_{1}(z)-\bar{z}$. Black triangles show zeros of $R_{1}^{\prime}(z)$ and white squares show the poles of $R_{1}(z)$. The picture shows that $R_{1}(z)-\bar{z}$ has three sense-preserving zeros (black disks in the bright region), and two sense-reversing zeros (black disks in the dark region).

Clearly, neither of the two sense-reversing zeros satisfies the conditions of Theorem 3.1. Denote by $z_{1}$ the leftmost sense-reversing zero of $R_{1}(z)-\bar{z}$; we have $\left|R_{1}^{\prime}\left(z_{1}\right)\right| \approx 0.5572$. Numerical experiments show that perturbing $R_{1}(z)-\bar{z}$ with the pole $\frac{\varepsilon_{1}}{z-z_{1}}$ results for all $\varepsilon_{1}>0$ small enough in a function that has four additional zeros nearby $z_{1}$. The result of such a perturbation is shown in Figure 5(b), The four additional zeros are explained by Theorem 3.14, and reducing $\varepsilon_{1}$ further does not result in further zeros in our numerical experiments.

Figure $5(\mathrm{c})$ shows the phase portrait of the function $R_{2}(z)-\bar{z}$, where $R_{2}(z)=R_{1}(z)+c$ with a deliberately chosen constant $c \in \mathbb{C}$ such that $R_{2}(z)-\bar{z}$ has a sense-reversing zero $z_{2}$ that coincides (numerically) with a zero of $R_{2}^{\prime}(z)=R_{1}^{\prime}(z)$ (leftmost zero of $R_{2}^{\prime}(z)$ ).

The effect of adding a pole at $z_{2}$ to $R_{2}(z)$, i.e., forming $R_{3}(z)=R_{2}(z)+$ $\frac{\varepsilon_{2}}{z-z_{2}}$, is shown in the phase portrait of $R_{3}(z)-\bar{z}$ in Figure $5(\mathrm{~d})$. An enlarged view on the perturbed region is given in Figure $5(\mathrm{e})$. We see that $R_{3}(z)-\bar{z}$ has 5 more zeros than $R_{2}(z)-\bar{z}$. Here $\varepsilon_{2}=4.5 \cdot 10^{-3}$, and we have chosen this value because a newly created zero of $R_{3}(z)-\bar{z}$ lies in the immediate vicinity of a zero of $R_{3}^{\prime}(z)$ (rightmost zero in Figure $5(\mathrm{e})$ ).

In order to obtain conditions that allow another application of Theorem 3.1. we could have tried to add another constant to $R_{3}(z)$ such that the conditions of the theorem are met. However, the rightmost zero of $R_{3}(z)-\bar{z}$, say, $z_{3}$, is already very close to a zero of $R_{3}^{\prime}(z)$. Figure $5(\mathrm{f})$ shows the effect of adding a pole at this position, i.e. setting $R_{4}(z)=R_{3}(z)+\frac{\varepsilon_{3}}{z-z_{3}}$ and considering the function $R_{4}(z)-\bar{z}$. Although the conditions of the theorem are not exactly met (we have $\left|R_{3}^{\prime}\left(z_{3}\right)\right| \approx 0.7936$ ), the same effect as in Figures $5(\mathrm{c}) 5(\mathrm{~d})$ can be observed. In particular, $R_{4}(z)$ is again extremal.

Notice also that it has been possible to choose the value $\varepsilon_{3}$ such that two newly created zeros of $R_{4}(z)-\bar{z}$ are very close to newly created zeros of $R_{4}^{\prime}(z)$, suggesting that it may be possible to repeat this perturbation procedure at least one more time.

\subsection{Extension to complex residues}

Our motivation for studying the effect of adding poles to $R(z)-\bar{z}$ comes from an astrophysical application in gravitational microlensing. This astrophysical application requires adding simple poles with positive residues. From a mathematical point of view, one may wonder about the effect of adding poles with complex residues on the number of (newly created) zeros. We will now give a brief and informal discussion of this case. 
Consider Rhie's function from (1), where we now replace $\varepsilon$ by $\varepsilon e^{i \theta}$, with $\varepsilon>0$ and $\theta \in \mathbb{R}$. Figure 6 shows phase portraits of $R_{\varepsilon e^{i \theta}}(z)$ centered at the origin for $n=3$ and several values of $\theta$. For positive perturbation $(\theta=0)$ the function $R_{\varepsilon}(z)-\bar{z}$ has $2 n=6$ zeros close to the origin (Figure 6(a)). We observe that with increasing argument, the zeros close to the origin approach each other (Figure 6(b) and finally vanish (Figures 6(c) and 6(d)) .

Heuristically this effect can be explained as follows. Let $z_{0}$ be a zero of $f(z)=R(z)-\bar{z}$, where $R(z)$ is rational with $\operatorname{deg}(R) \geq 2$. Consider the perturbed function $F(z)=f(z)+\frac{\varepsilon}{z-z_{0}}$. This function can be approximated (after translation $w:=z-z_{0}$ ) by the function $G(w)$ in (5). The zeros of $G(w)$ close to $z_{0}$ give rise to zeros of $F(z)$; see Sections 3.1 and 3.3 . The zeros of $G(w)$ are the solutions of the equation

$$
c w^{n}+\varepsilon-|w|^{2}=0 .
$$

For small values of $w$, this equation is approximated by $\varepsilon-|w|^{2}=0$, which has solutions only for $\varepsilon \geq 0$. This suggests that $F(z)$ has no zeros near $z_{0}$ for sufficiently small $\varepsilon$ chosen "away from the positive real axis".

\subsection{Extension to poles of higher order}

So far we considered perturbations by simple poles. One may wonder about the effects of adding poles of higher order. We give a brief and informal discussion of this case.

For simplicity, let $f(z)$ be as in Theorem 3.14. Suppose $z_{0} \in \mathbb{C}$ is not a pole of $f(z)$ and consider the function $F(z)=f(z)+\frac{\varepsilon}{\left(z-z_{0}\right)^{k}}$, where now $k \geq 1$, and assume that $F(z)$ is regular as well. As before, we can show that on a sufficiently large circle $\Gamma$ we have $V(f ; \Gamma)=V(F ; \Gamma)$. Further, there exists a circle $\gamma$ centered at $z_{0}$, not containing any zero or pole of $f(z)$ (except possibly $z_{0}$ ), such that for sufficiently small $\varepsilon$ the functions $f(z)$ and $F(z)$ have the same number of zeros with same indices outside $\gamma$, and that $V(f ; \gamma)=V(F ; \gamma)$ holds. Let us denote by $n_{+}^{\text {new }}$ and $n_{-}^{\text {new }}$ the number of sense-preserving and sense-reversing zeros of $F(z)$ inside $\gamma$, respectively. By the argument principle (Theorem 2.6) we then have

$$
\operatorname{ind}\left(z_{0} ; f\right)=V(f ; \gamma)=V(F ; \gamma)=-k+n_{+}^{\text {new }}-n_{-}^{\text {new }},
$$

or, equivalently, $n_{+}^{\text {new }}-n_{-}^{\text {new }}=k+\operatorname{ind}\left(z_{0} ; f\right)$. Thus the total number of zeros of the regular function $F(z)$ inside $\gamma$ is

$$
n_{+}^{\text {new }}+n_{-}^{\text {new }}=k+\operatorname{ind}\left(z_{0} ; f\right)+2 n_{-}^{\text {new }} \geq k+\operatorname{ind}\left(z_{0} ; f\right) .
$$

We illustrate this result in Figure 7. In Figure 7(a), the same function as used in Section 4.3 is shown; the indicated points correspond to a sensepreserving zero $\left(\operatorname{ind}\left(z_{1} ; f\right)=1\right)$, a sense-reversing zero $\left(\operatorname{ind}\left(z_{2} ; f\right)=-1\right)$ and a non-exceptional point $\left(\operatorname{ind}\left(z_{3} ; f\right)=0\right)$. 
Adding a pole of order $k=4$ with residue $\varepsilon=10^{-5}$ at the designated positions $z_{1}, z_{2}$ and $z_{3}$ results in 5,3 , and 4 new solutions nearby the position of the pole, which is shown in Figures $7(\mathrm{~b}) 7(\mathrm{~d})$. In each case, the minimum number of zeros are created (see the bound (13)), and $n_{-}^{\text {new }}=0$.

We performed extensive numerical experiments for various combinations of $k$, index of $z_{0}$ and the number of vanishing derivatives at $z_{0}$. In each case, if $\varepsilon$ was chosen sufficiently small, the minimum number of zeros was created and $n_{-}^{\text {new }}=0$. Further, the effect of complex residues $\varepsilon$ we observed was significantly different from the situation in Section 4.5 , the created zeros are merely rotated according to the value of $\arg (\varepsilon)$.

\section{Conclusions and Outlook}

We have generalized Rhie's technique [19] for constructing extremal point lenses to arbitrary rational harmonic functions of the form $f(z)=R(z)-\bar{z}$. We have shown that if $f\left(z_{0}\right)=0$ and the first $n-2$ derivatives of $R(z)$ vanish at $z_{0}$, while $R^{(n-1)}\left(z_{0}\right) \neq 0$, then the function $f(z)+\frac{\varepsilon}{z-z_{0}}$ has at least $2 n$ zeros near the circle $\left|z-z_{0}\right|=\sqrt{\varepsilon}$, provided $\varepsilon>0$ is sufficiently small. In particular, our general results cover Rhie's original construction; see Corollary 3.3 . We also gave lower bounds on the number of additional zeros if $f(z)$ is perturbed by a pole at any other point $z_{0} \in \mathbb{C}$.

We have briefly studied extensions of our results to complex residues (Section 4.5) and poles of higher order (Section 4.6) by means of examples, but we did not pursue these directions with full rigor.

Theorems 3.1 and 3.14 give sufficient conditions for lower bounds on the number of newly created zeros in the vicinity of the point where the pole is added, which raises two open questions. Firstly, the examples in Section 4.2 demonstrate that the stated conditions are not necessary for the creation of the stated number of zeros. They show that more images can be created if the perturbation residue $\varepsilon$ is chosen somewhat "too large". It would be interesting to quantify this effect. Secondly, we believe that for sufficiently small perturbations, no more than the stated number of zeros are created; see also Remark 3.8 . We have performed extensive numerical experiments that support this claim. However, a rigorous analysis is yet to be done.

Acknowledgements We would like to thank Elias Wegert for comments on the manuscript and creating the color scheme used in the illustrations. We are grateful to the anonymous referee for carefully reading the manuscript and for giving us many useful suggestions.

The work of R. Luce was supported by Deutsche Forschungsgemeinschaft, Cluster of Excellence "UniCat". 


\section{References}

[1] Mark Benevich Balk. Polyanalytic Functions. Mathematical Research. 63. Berlin: Akademie-Verlag. 197 p. , 1991.

[2] Johann Bayer and Charles C. Dyer. Maximal lensing: mass constraints on point lens configurations. Gen. Relativity Gravitation, 39(9):14131418, 2007.

[3] Johann Bayer and Charles C. Dyer. Erratum: Maximal lensing: mass constraints on point lens configurations. Gen. Relativity Gravitation, 41(3):669, 2009.

[4] Pavel M. Bleher, Youkow Homma, Lyndon L. Ji, and Roland K. W. Roeder. Counting zeros of harmonic rational functions and its application to gravitational lensing. International Mathematics Research Notices, 2014(8):2245-2264, 2014.

[5] John B. Conway. Functions of one complex variable, volume 11 of Graduate Texts in Mathematics. Springer-Verlag, New York, second edition, 1978.

[6] Peter Duren. Harmonic mappings in the plane, volume 156 of Cambridge Tracts in Mathematics. Cambridge University Press, Cambridge, 2004.

[7] Peter Duren, Walter Hengartner, and Richard S. Laugesen. The argument principle for harmonic functions. Amer. Math. Monthly, 103(5):411-415, 1996.

[8] Lukas Geyer. Sharp bounds for the valence of certain harmonic polynomials. Proc. Amer. Math. Soc., 136(2):549-555, 2008.

[9] I. Glicksberg. A remark on Rouché's theorem. Amer. Math. Monthly, 83(3):186-187, 1976.

[10] Peter Henrici. Applied and computational complex analysis. WileyInterscience [John Wiley \& Sons], New York, 1974. Volume 1: Power series - integration - conformal mapping - location of zeros, Pure and Applied Mathematics.

[11] Dmitry Khavinson and Genevra Neumann. On the number of zeros of certain rational harmonic functions. Proc. Amer. Math. Soc., 134(4):1077-1085 (electronic), 2006.

[12] Dmitry Khavinson and Genevra Neumann. From the fundamental theorem of algebra to astrophysics: a "harmonious" path. Notices Amer. Math. Soc., 55(6):666-675, 2008. 
[13] Dmitry Khavinson and Grzegorz Świątek. On the number of zeros of certain harmonic polynomials. Proc. Amer. Math. Soc., 131(2):409-414 (electronic), 2003.

[14] S.-Y. Lee, A. Lerario, and E. Lundberg. Remarks on Wilmshurst's theorem. ArXiv e-prints, August 2013.

[15] Jörg Liesen. When is the adjoint of a matrix a low degree rational function in the matrix? SIAM J. Matrix Anal. Appl., 29(4):1171-1180, 2007.

[16] Robert Luce, Olivier Sète, and Jörg Liesen. Sharp parameter bounds for certain maximal point lenses. Gen. Relativity Gravitation, 46(5):46:1736, 2014.

[17] S. Mao, A. O. Petters, and H. J. Witt. Properties of Point Mass Lenses on a Regular Polygon and the Problem of Maximum Number of Images. In T. Piran and R. Ruffini, editors, Recent Developments in Theoretical and Experimental General Relativity, Gravitation, and Relativistic Field Theories, page 1494, 1999.

[18] A. O. Petters and M. C. Werner. Mathematics of gravitational lensing: multiple imaging and magnification. Gen. Relativity Gravitation, 42(9):2011-2046, 2010.

[19] S. H. Rhie. n-point Gravitational Lenses with 5(n-1) Images. ArXiv Astrophysics e-prints, May 2003.

[20] T. Sheil-Small. Complex polynomials, volume 75 of Cambridge Studies in Advanced Mathematics. Cambridge University Press, Cambridge, 2002.

[21] T. J. Suffridge and J. W. Thompson. Local behavior of harmonic mappings. Complex Variables Theory Appl., 41(1):63-80, 2000.

[22] Elias Wegert. Visual complex functions. Birkhäuser/Springer Basel AG, Basel, 2012. An introduction with phase portraits.

[23] Elias Wegert and Gunter Semmler. Phase plots of complex functions: a journey in illustration. Notices Am. Math. Soc., 58(6):768-780, 2011.

[24] A. S. Wilmshurst. The valence of harmonic polynomials. Proc. Amer. Math. Soc., 126(7):2077-2081, 1998. 


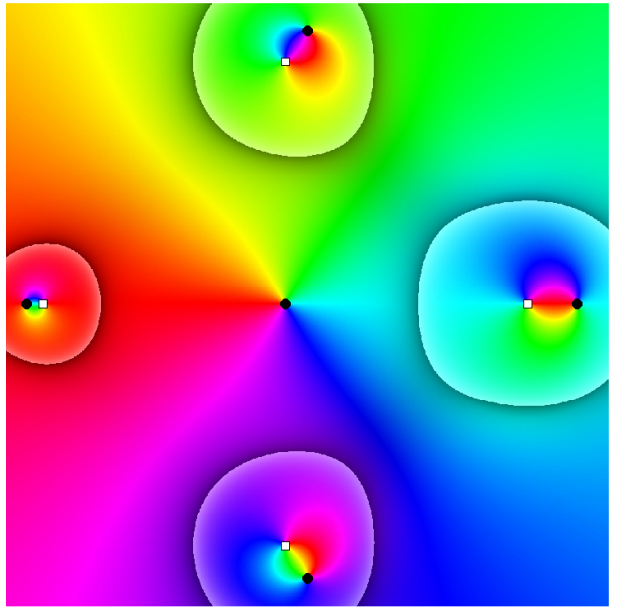

(a) Unperturbed function

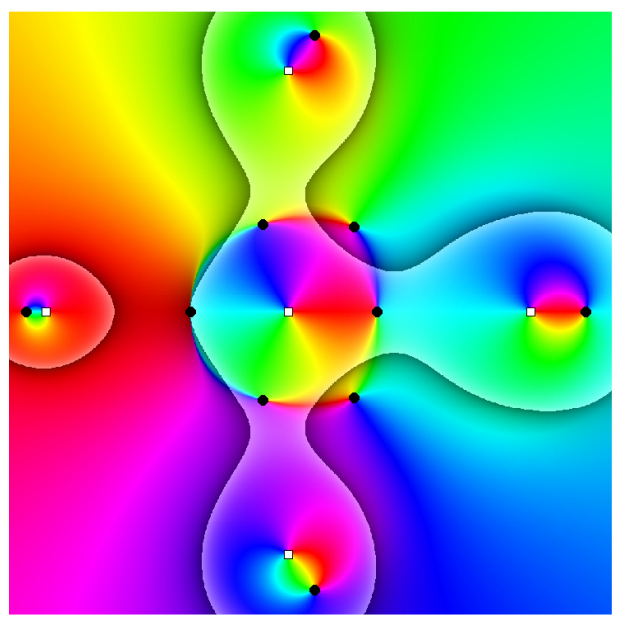

(c) $\varepsilon=0.05$

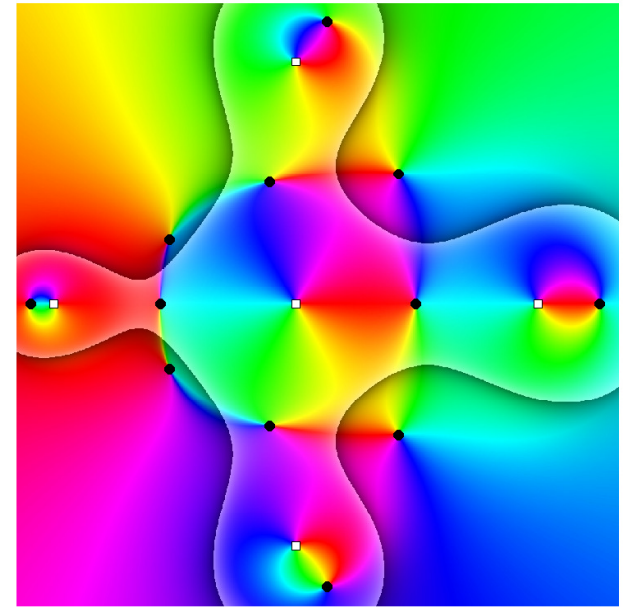

(b) $\varepsilon=0.1$

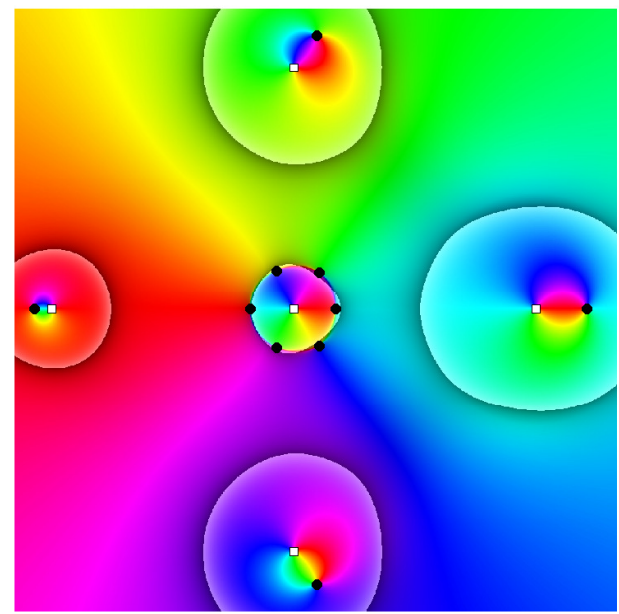

(d) $\varepsilon=0.01$

Figure 3: More than $2 n$ additional zeros may occur; see Section 4.2 . 

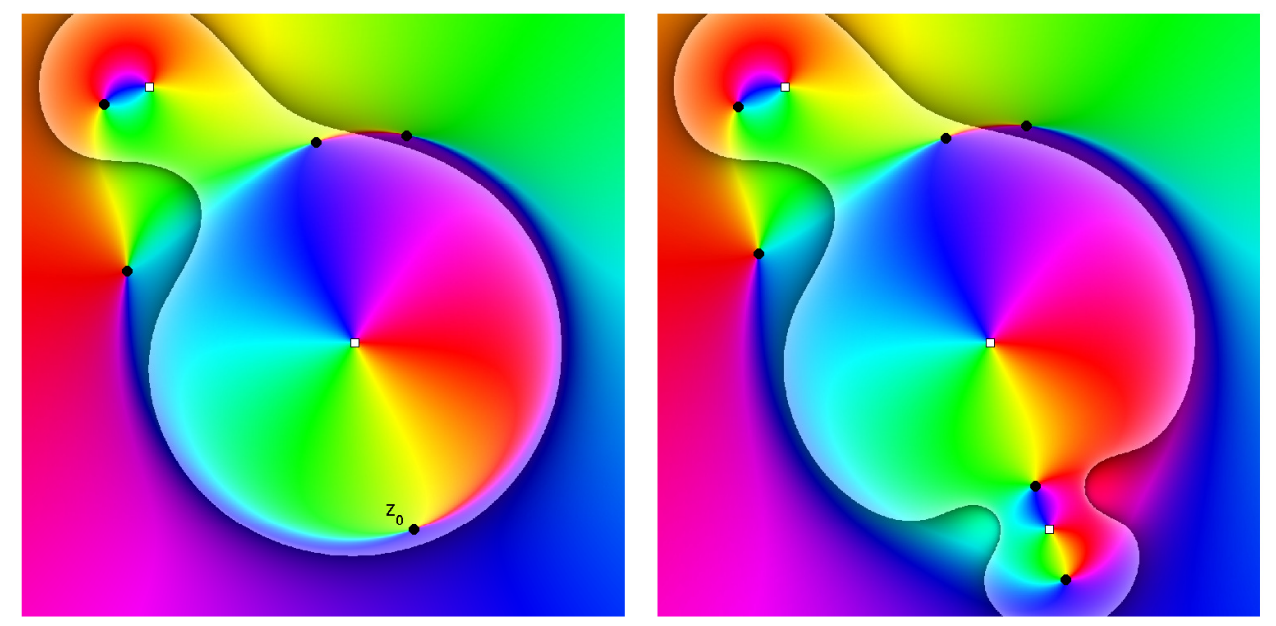

Figure 4: Perturbation at a sense-preserving zero; see Section 4.3 . 


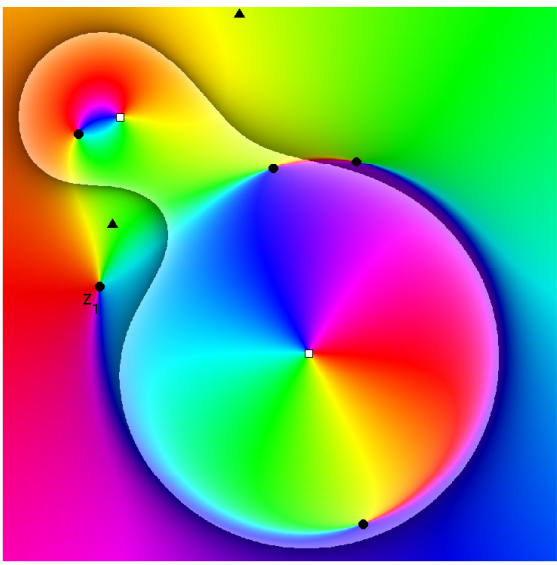

(a)

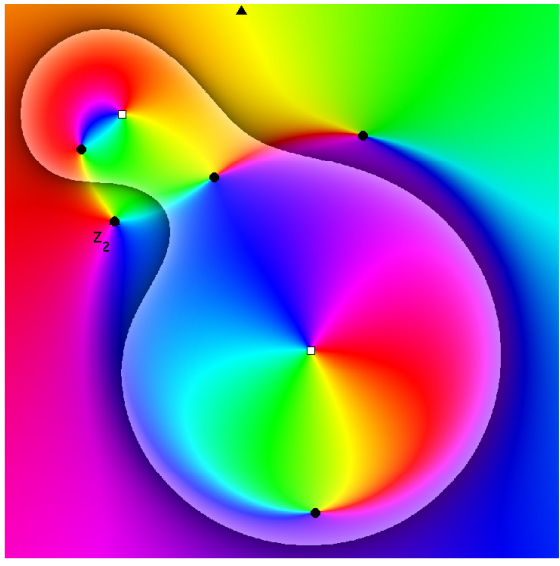

(c)

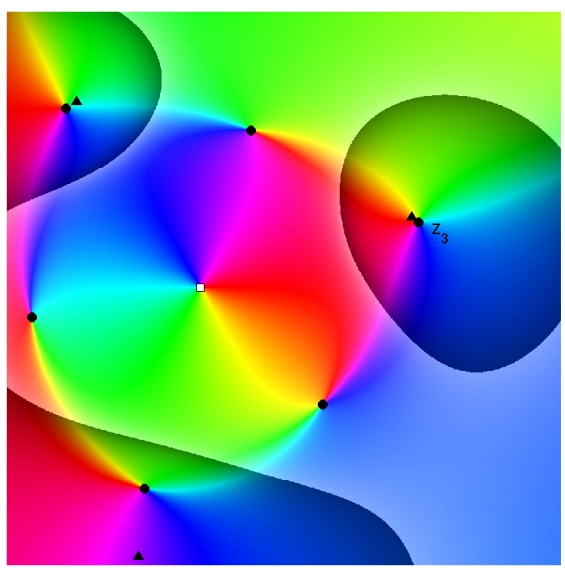

(e)

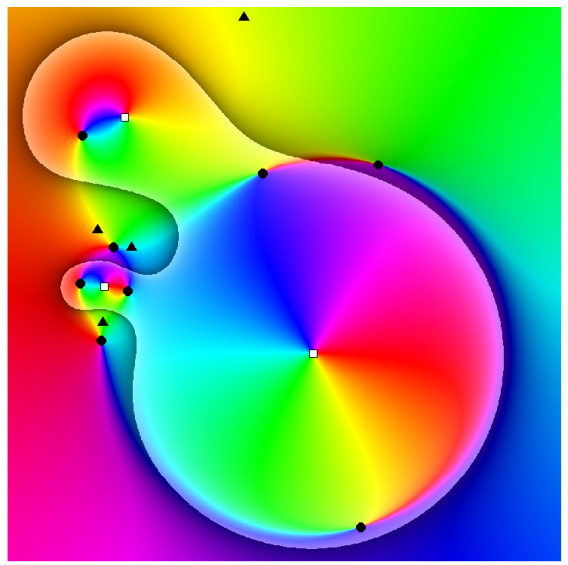

(b)

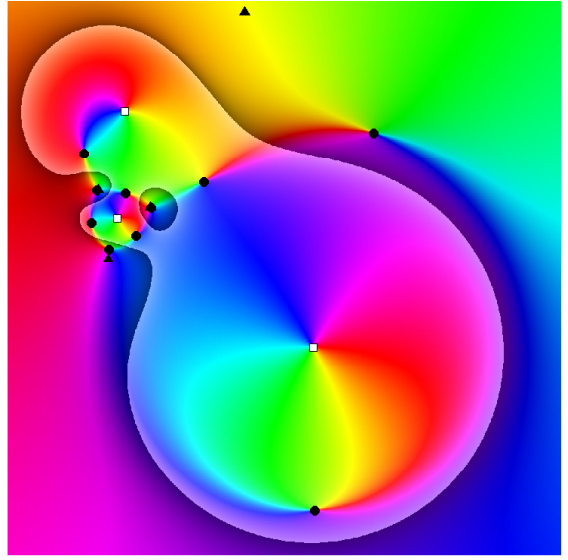

(d)

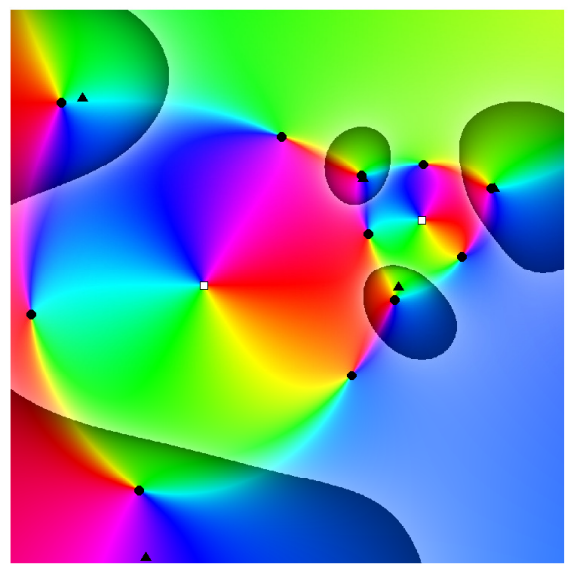

(f)

Figure 5: Iterative perturbation of a random rational function; see Section 4.4. Black triangles indicate the zeros of $R^{\prime}(z)$. 




(a) $\theta=0$ (positive perturbation)

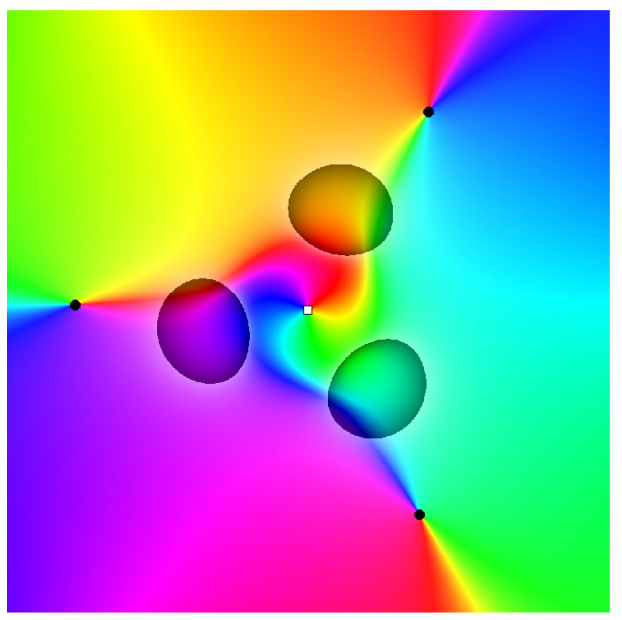

(c) $\theta=0.2 \pi$

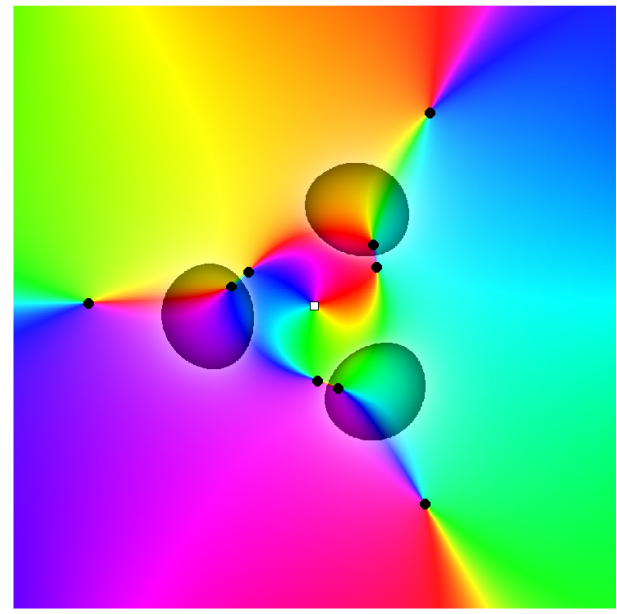

(b) $\theta=0.1 \pi$

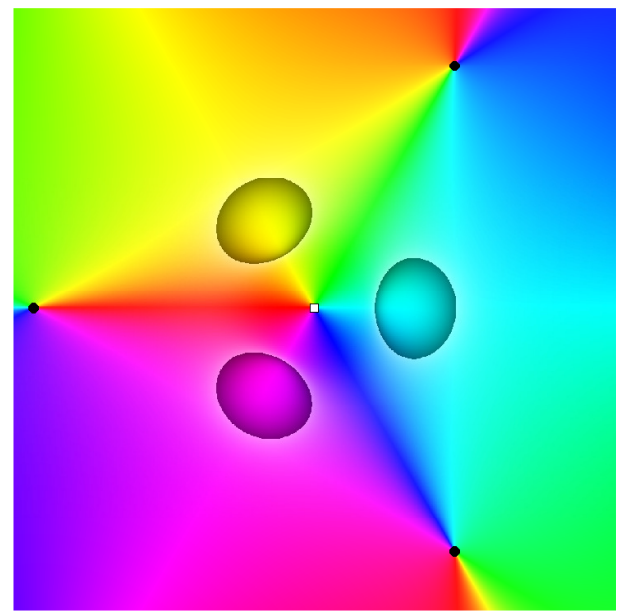

(d) $\theta=\pi$ (negative perturbation)

Figure 6: Phase portraits of Rhie's function $R_{\varepsilon}(z)$ from (1) with $\varepsilon>0$ replaced by $\varepsilon e^{i \theta}$; see Section 4.5 


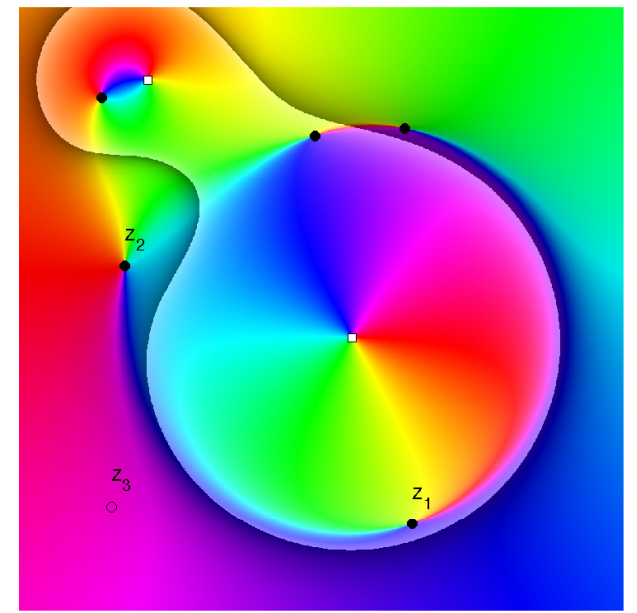

(a) Unperturbed function.

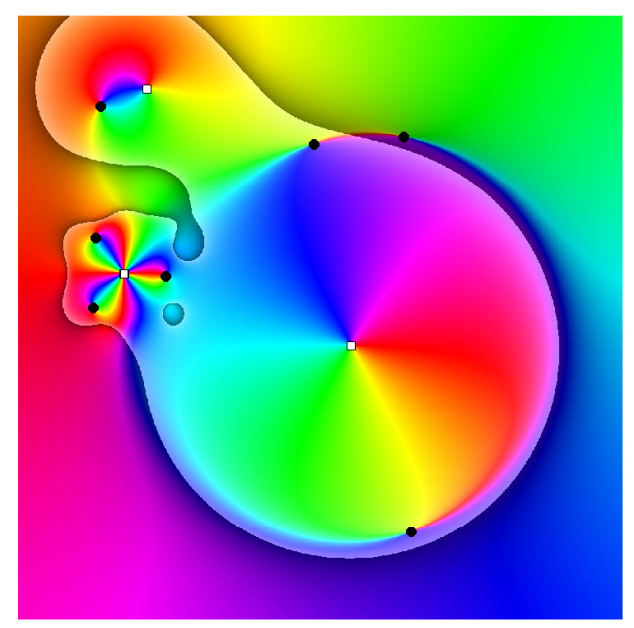

(c) Perturbing a sense-reversing zero.

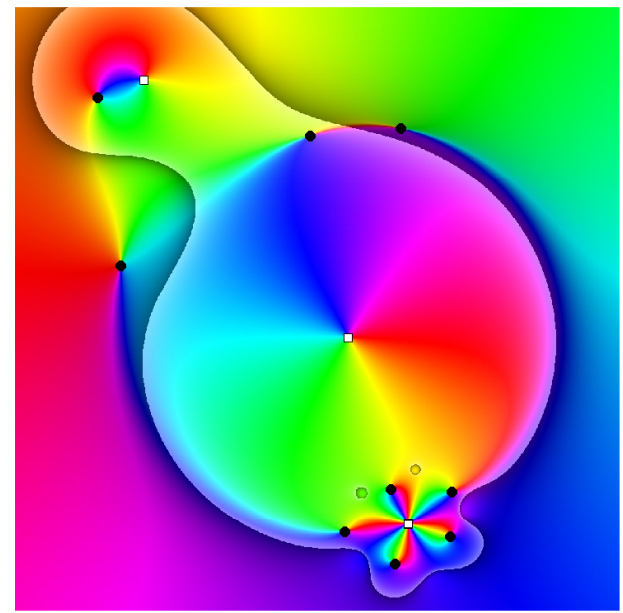

(b) Perturbing a sense-preserving zero.

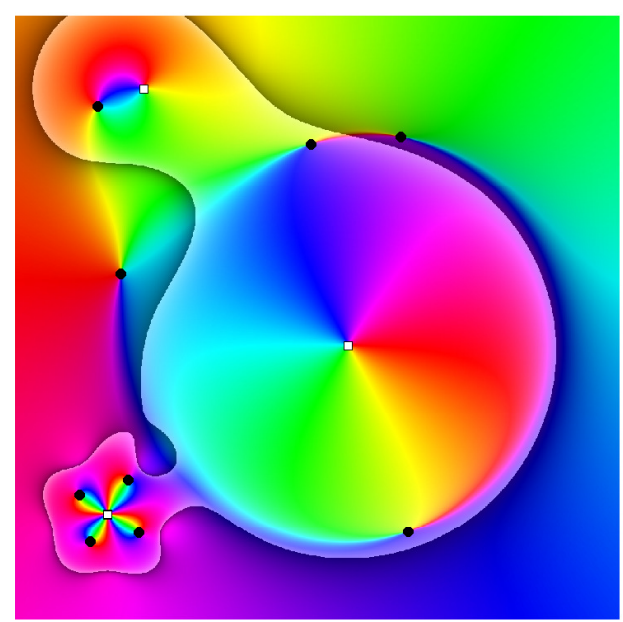

(d) Perturbing a non-zero.

Figure 7: Perturbations with poles of higher order; see Section 4.6 . 